\title{
ANÁLISIS DE MUERTES POR CAUSAS EXTERNAS EN EL DEPARTAMENTO DE BOLÍVAR: ENERO - DICIEMBRE 2011-2015 ${ }^{1}$ Analysis of deaths by external causes in the department of Bolivar January - December 2011-2015
}

\section{Carlos Alberto Almanza Agámez $^{2}$}

Fecha de Recepción: 7 de abril de 2016

Fecha de Aceptación: 20 de mayo de 2016

SUMARIO: 1. Introducción; 2. Marco Conceptual; 3. Aspectos Metodológicos; 4.Resumen Ejecutivo: Enero - Diciembre Del 2015 En Comparación con mismo periodo 2011-2014; 5. Homicidio; 6. Accidentes De Tránsito; 7. Muertes No Intencionales; 8. Suicidios;

9. Conclusiones; 10. Referencias bibliográficas

\footnotetext{
${ }^{1}$ Este artículo de investigación fue elaborado al interior del grupo de investigación Derecho Público, avalado y financiado por la Corporación Universitaria Rafael Núñez, IES de origen privado. La recopilación de información fue financiada por el Departamento de Bolívar, que patrocina el funcionamiento de COISBOL.

${ }^{2}$ ALMANZA AgÁMEZ, CARLOS AlBeRTo. Profesional del Derecho, Especialista en Estudios Políticos - Económicos, Especialista en Derecho Administrativo. Maestrante en Derecho. Docente Universitario e Investigador. Decano Facultad de Ciencias Sociales y Humanas - Corporación Universitaria Rafael Núñez. Asesor Académico COISBOL durante el año 2015. CvLac: http://scienti1. colciencias.gov.co:8081/cvlac/visualizador/generarCurriculoCv.do?cod_rh=0000909424
} 


\section{CÓMO SE CITA ESTE ARTÍCULO (Normas APA-6)}

Almanza Agámez, Carlos Alberto (2016). Análisis de muertes por causas externas en el departamento de Bolívar: Enero - diciembre 2011-2015. Revista Jurídica Mario Alario D’Filippo, VIII (16), pág 12-37.

\section{RESUMEN}

Se analiza la evolución y el comportamiento de las variables que integran las muertes por causas externas MCE en el territorio del Departamento de Bolívar durante el periodo enero a diciembre de los años 2011 a 2015, que corresponde al periodo de gobierno del Gobernador Juan Carlos Gossain, y un año antes. El análisis de Las Muertes por Causas Externas - MCE-, permite identificar una visión histórica del periodo abarcado, que permite generar insumos objetivos para evaluar la efectividad y eficacia de las políticas en materia de seguridad y convivencia desarrolladas interinstitucionalmente, y de ser necesario introducir mejoras en las mismas para periodos de gobierno sucesivos. Las causas a analizar son homicidios, accidentes de tránsito, muerte no intencional y suicidio, con sus respectivas tasas de mortalidad.

\section{PALABRAS CLAVE}

MCE, homicidio, accidente tránsito, muerte no intencional, suicidio, tasa de mortalidad.

\section{ABSTRACT}

The following text analyzes the evolution and the behavior of the variables that make up deaths by external causes ( DEC) in the territory of the Department of Bolivar during the period of January to December from 2011 - 2015 , corresponding to the term in office of Governor Juan Carlos Gossain as well as one year before his term. The deaths caused by external causes (DEC) having been analyzed permits to identify a historic vision of the period covered. Doing so it permits to evaluate the effectiveness and efficiency of policies developed security and improvement of the lifestyle for the community and various institutions, and if necessary improvements are made thereto for successive periods of government. The topics and causes that will be analyzed are the following: homicide, traffic accidents, unintentional deaths, suicide along with the corresponding mortality rates.

\section{KEYWORDS}

DEC, homicide, traffic accidents, unintentional deaths, suicide, mortality rates. 


\section{INTRODUCCIÓN}

De conformidad con el Decreto 353 del 26 de Junio del 2012 de la Gobernación de Bolívar, el Centro de Observación e Investigación Social del Departamento de Bolívar COISBOL, cumple una función misional orientada a generar información técnica sobre el comportamiento de los hechos delictivos y la violencia del Departamento de Bolívar. Su objetivo general es aumentar el conocimiento y el análisis existente sobre la falta de convivencia y la inseguridad ciudadana a través de la generación de información consolidada, confiable y oportuna, que permita el estudio de las tendencias e identificación de las zonas críticas para la formulación y priorización de políticas, planes y programas que sean útiles y pertinentes para enfrentar la problemática identificada y garantizar los derechos de los ciudadanos.

Es preciso anotar que COISBOL se encuentra adscrito a la Dirección de Seguridad y convivencia Ciudadana de la Gobernación de Bolívar, y Él mismo coordina un "Comité Interinstitucional de Validación y Concertación de la Información" que recopila información primaria sobre las muertes por causas externas en el Departamento, proveída por las siguientes entidades:

1. Armada Nacional

2. Cuerpo Técnico De Investigación (CTI)

3. Fiscalía General De La Nación

4. Instituto Nacional De medicina Legal

5. Policía Del Departamento De Bolívar - DEBOL.

6. SIJIN DEBOL

7. Policía Metropolitana De Cartagena

8. Policía Del Magdalena Medio

9. Migración Colombia

En este marco se presenta el siguiente análisis pormenorizado de los datos relevantes que tuvieron incidencia en la seguridad y la convivencia ciudadana en el departamento de Bolívar, enfocados en las muertes por causas externas - MCE -, esto es, aquellas que no se producen en forma natural, desagregadas en cuatro variables a tratar: homicidios, muertes por accidentes de tránsito, suicidios, y muertes no intencionales. Todas ellas se caracterizan por ser muertes violentas que lesionan el principal bien jurídico en cabeza de todas las personas, tutelado por la Constitución Política de 1991 y el Estado como es la Vida ${ }^{3}$ El análisis se limita a observar el comportamiento y variación anual comparando

\footnotetext{
${ }^{3}$ Tanto para Medicina Legal, como para el DANE, se entiende por Muertes Violentas aquellas que ocurren a consecuencias de una causa externa, que se produce por un agente agresor externo y genera en la victima una lesión mortal. Integran el grupo de las causas externas, los homicidios, suicidios, los accidentes de tránsito, y otros accidentes como caídas desde su propia altura, electrocuciones, quemaduras, mordeduras, ahogamientos, envenenamientos, entre otros. Véase: DANE. (2010). Preguntas relacionadas con las muertes violentas. Preguntas Frecuentes. (pp. 10-11). Recuperado de: https://www.dane.gov.co/files/investigaciones/poblacion/ preguntas_frecuentes.pdf
} 
todos los meses objeto de indagación, esto es enero a diciembre de 2011 a 2015, su distribución por Zodes, y desagregación por municipios. El lector que desee indagar un poco más en profundidad podrá solicitar a COISBOL a efectos de poder detallar en el informe completo, atendiendo a otras variables que no se exponen en el presente informe.

\section{MARCO CONCEPTUAL}

- MUERTES POR CAUSAS EXTERNAS (MCE): Las MCE son aquellas que no se producen de forma natural. Estas se desagregan en cuatro variables a tratar:

1. Homicidios.

2. Accidentes de tránsito.

3. Suicidios.

4. No Intencionales.

- LA TASA DE MORTALIDAD POR CAUSA EXTERNA Es el número total de defunciones estimadas por homicidios, accidentes de tránsito, muertes no intencionales y suicidios en una población dividido por el total de esa población, expresada por cada cien mil habitantes, en adelante PCCMH. Metodología de cálculo: MLCExt $=\left(\mathrm{N}^{\circ}\right.$ total de defunciones estimadas por lesiones de causa externa en una población $/ \mathrm{N}^{\circ}$ total de población ) x 100.000 .

- HOMICIDIO: Privación de la vida de un ser humano por la acción de otro, ya sea dolosa o culposamente, convirtiéndose así en uno de los delitos más graves. Este se encuentra tipificado en la legislación penal, Ley 599 de Julio 24 del 2000, dentro de la Parte Especial referida a "De los delitos en particular" en su Título I "Delitos contra la vida y la integridad personal" y en el Capítulo Segundo "Del homicidio", y legislación complementaria.

- MUERTES POR ACCIDENTES DE TRANSITO (MACCT): Es el suceso imprevisto producido por la participación de un vehículo o más en las vías o carreteras, ocasionando la muerte a personas. Para la Organización Panamericana de la Salud, las Muertes por Accidente de Tránsito, para que sean considerada como tal, deben ocurrir dentro de los treinta (30) días posteriores al hecho que la ocasiona ${ }^{4}$

- MUERTES NO INTENCIONALES: Los fallecimientos "absurdos" -como los que ocurren por caerse de la cama, sumados a los ocasionados de manera involuntaria como los accidentes en el hogar y en el trabajo- tienen la tendencia a ser la tercera causa de muerte en el país, después de los homicidios y los accidentes de tránsito.

${ }^{4}$ Organización Panamericana de la Salud (2009). Informe sobre el estado de la seguridad vial en la región de las Américas. Washington, D.C.: Organización Panamericana de la Salud, p. 9. Recuperado de: http://www.who.int/violence_injury_prevention/road_safety_ status/2009/gsrrs_paho.pdf 
- SUICIDIOS: Es la acción de quitarse la vida por un acto voluntario y violento. Acción por la cual una persona acaba con su propia vida. Se clasifica como conducta autodestructiva porque esa acción puede conducir directamente a la muerte o puede ser un intento, pero siempre existe una acción letal en su misma esencia.

\section{ASPECTOS METODOLÓGICOS}

Los resultados de la investigación socio-jurídica aquí expuestos están metodológicamente fundamentados en un diseño de estudio descriptivo, de corte transversal poblacional para Bolívar (excluye al Distrito de Cartagena y sus corregimientos) correspondiente al año 2015. El periodo objeto de análisis es el comprendido entre enero a diciembre del 2015, tomando como referencia para análisis comparativo los mismos periodos entre los años 2011 a 2015.

Este informe cuantitativo, además de exponer los resultados de un proceso de investigación socio-jurídica, tiene como utilidad servir como insumo a las autoridades políticoadministrativas, policías y militares, con jurisdicción en el Departamento de Bolívar, para generar procesos y estrategias de intervención que promuevan la convivencia y seguridad ciudadana, además de suministrar datos para realizar seguimiento y evaluación de las distintas políticas que se implementan en el departamento sobre conflictividad, seguridad, convivencia y aspectos sociales, entre otros objetivos específicos señalados en el Art. 3ro del Decreto 353 del 2012 de la Gobernación de Bolívar.

Para la elaboración de este informe se tiene como fuente las actas mensuales de los comités interinstitucionales de validación y concertación de muertes por causas externas -MCE-, coordinados por COISBOL, que a su vez se nutren de:

- Reportes de necropsias emitidos por la Regional Norte del Instituto Nacional de Medicina Legal y Ciencias Forenses- Seccional Bolívar

- El sistema Penal Oral Acusatorio (SPOA) de la Fiscalía General de la Nación (incluyendo al CTI).

- Armada Nacional.

- Datos suministrados por el Centro de Investigaciones Criminológicos de la Policía Nacional a través de sus unidades, así:

- Departamentos de Policía Bolívar DEBOL con 34 municipios

- Departamento de Policía de Magdalena Medio, DEMAM, con 6 municipios

- Policía Metropolitana de Cartagena MECAR con 5 municipios (Excluyendo al Distrito de Cartagena, que corresponde a datos del Centro de Observación y Seguimiento del Delito, COSED, y es objeto de seguimiento por parte de las autoridades distritales).

- Migración Colombia.

Vale anotar que la información que consolida el Comité Interinstitucional de Validación y Concertación de la Información hace parte de una labor que busca también motivar y fortalecer el trabajo institucional, coordinado entre las autoridades político- 
administrativas, la policía Nacional y demás agencias de seguridad y justicia del Estado en el territorio del Departamento de Bolívar. Todo, bajo los principios de oportunidad, confidencialidad, objetividad y legalidad que rigen la labor de $\mathrm{COISBOL}^{5}$

Según la Resolución No. 008430 de 1993 del Ministerio de Salud y Protección Social, la investigación se clasifica como "Investigación con riesgo menor que mínimo", que no requiere consentimiento informado y no fue presentado a Comité de Ética. Se preservó el anonimato de las víctimas y la respectiva reserva del proceso penal.

\section{RESUMEN EJECUTIVO: ENERO - DICIEMBRE DEL 2015 EN COMPARACIÓN CON MISMO PERIODO 2011-2014}

La evolución y el comportamiento de las variables que integran las muertes por causas externas MCE en el territorio del departamento de Bolívar permite identificar una visión histórica del mismo que permita evaluar la efectividad y eficacia de las políticas en materia de seguridad y convivencia desarrolladas interinstitucionalmente, y de ser necesario introducir mejoras en las mismas.

\section{Gráfica 1: TOTAL DE MUERTES POR CAUSAS EXTERNAS ENERO - DICIEMBRE COMPARATIVO 2011 - 2015}

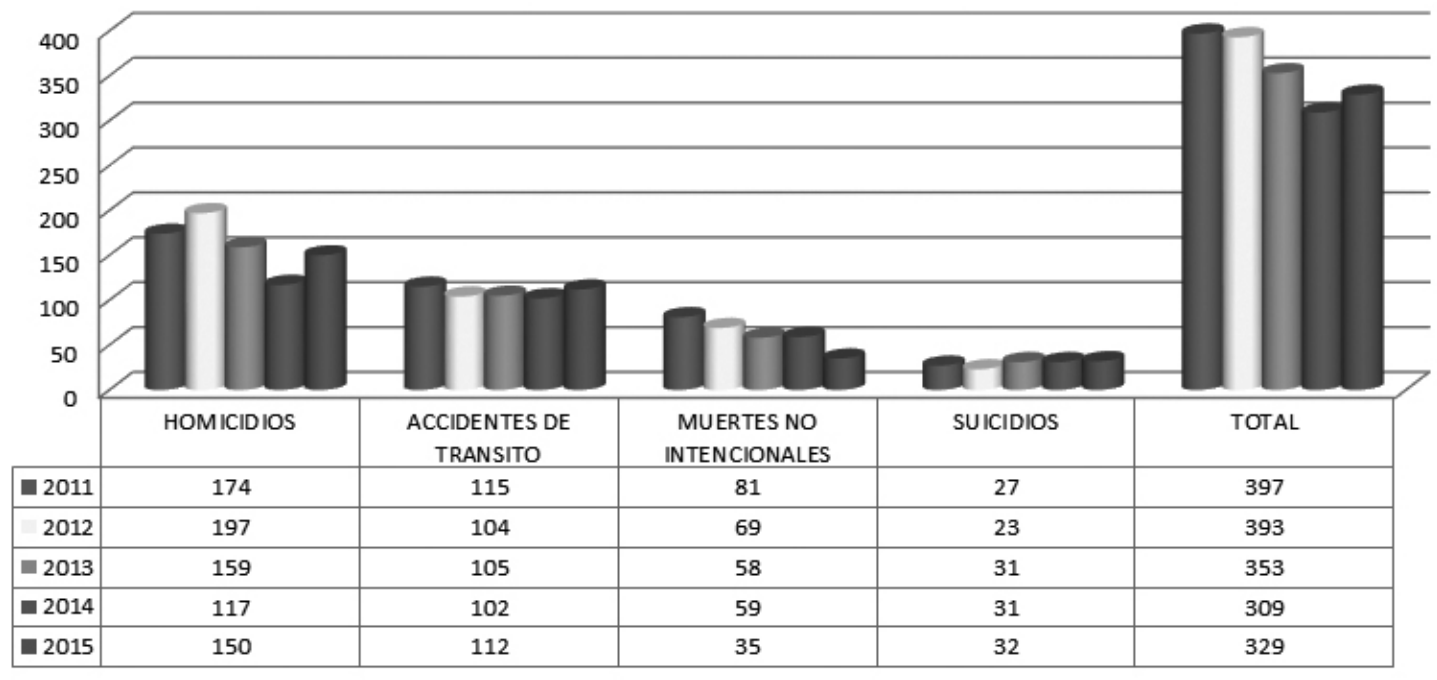

Fuente: Comité de Validación MCE - COISBOL.

\footnotetext{
${ }^{5}$ El Decreto 353 del 26 de Junio del 2012 en su artículo 4 contempla que el Centro de Observación Social e Investigación de Bolívar se regirá por los siguientes principios:

-Oportunidad: La información se recopila, analiza y reporta en el momento adecuado de tal manera que se puedan implementar acciones de prevención y control de una determinada conducta conflictiva, violenta o delictual.

- Confidencialidad: Se guardará discreción y reserva sobre los documentos, hechos e informaciones a los cuales el Centro de Observación e Investigación Social de Bolívar "COISBOL", tenga acceso o conocimiento como consecuencia del ejercicio de sus labores. - Objetividad: El análisis de la información se hace con rigurosidad metodológica de tal manera que sus resultados reflejen de la mejor manera posible la realidad observada.

- Legalidad: Las propuestas y estudios de política pública realizados desde "COISBOL" se hacen en el marco del Estado social de derecho, respetando condiciones de igualdad, sin discriminación por razones de sexo, raza, origen nacional, familiar, lengua, religión o de opinión política o filosófica.
} 
Lo primero en observarse es que en el periodo enero - diciembre del 2015 -a excepción de las muertes por accidente de tránsito y suicidios- sigue mostrando una dinámica favorable frente al mismo periodo de los años precedentes. El 2015 ha sido, en términos globales, el segundo periodo objeto de análisis con menos casos de muertes ( 329 en total a diciembre), 28 casos más que en el año 2014, aunque sigue siendo inferior a los años precedentes, 2013 hacía atrás. Esta reducción es significativa si se compara con el año previo al Gobierno del Bolívar Ganador, año 2011, que presentó un total de 397 casos de MCE a diciembre, de forma tal que a 2015 se observa una reducción del 17,12\%. Siendo el promedio del periodo analizado de 356 casos ( 1781 casos en 5 años), el 2015 se ubica por debajo del promedio comparado (329 casos), por lo que esta dinámica fue consolidando a Bolívar y sus municipios como un territorio con mejoría en sus índices de seguridad y disminución de los MCE.

Respecto al homicidio, si bien la política de Bolívar Seguro muestra que se mantuvo un promedio de casos por debajo de los últimos cinco años -lo que de por si sigue siendo un logro destacable-, no puede obviarse el hecho de que, frente al año inmediatamente anterior, a Diciembre de 2014, hubo un incremento de casos que rompió la tendencia a disminuir que se venía consolidando entre 2013 (159 casos), y 2014 (el periodo más favorable con 117 casos). Los 150 casos de muerte por homicidio en Bolívar durante enerodiciembre del 2015 siguen estando por debajo, en todo caso, del promedio del periodo comparado, que es de 159,4 casos promedio en los últimos cinco años (2011-2015).

La variable accidentes de tránsito es en el periodo analizado del 2015 la segunda variable de muertes por causas externas en el departamento de Bolívar. 112 casos presentados (a diciembre de 2015) rompieron la muy leve tendencia a la baja, durante el tiempo de análisis. De 538 casos en total, de 2011 a 2015, el promedio anual es de 105,6 casos aproximadamente, de forma tal que el 2015 con 107,6 casos presenta un ligero decrecimiento de la accidentalidad con resultados fatales en términos de vida, muy a pesar de los reportes que la fuerza pública y demás instancias institucionales informan sobre los constantes operativos y medidas tomadas para prevenir accidentes en las carreteras del departamento y sus municipios.

El reporte de muertes no intencionales es el que mejor indicadores muestra, con solo 35 casos, muy por debajo del promedio del periodo comparado, que es de 60,4 casos. Es notoria la mejoría con el año inmediatamente anterior, disminuyendo de los 59 casos del 2014 a solo 35 en 2015 (disminuyó del 2014 a 2015 en un 59,32\%).

Por último, el suicidio llama la atención (32 casos), no solo porque es levemente superior al número de casos del 2014 (31 casos), sino que además supera el promedio del periodo 2011-2015 comparado (28,8 promedio anual en los últimos cinco 5 años). En el periodo analizado (a diciembre del 2015) se presentó un caso más de los ocurridos en el año inmediatamente anterior. 
A continuación se observa el comportamiento de MCE entre 2011 y 2015 periodo enero a diciembre del 2015:

\section{Gráfica 2: COMPORTAMIENTO TOTAL DE MUERTES POR CAUSAS EXTERNAS}

ENERO - DICIEMBRE COMPARATIVO 2011 - 2015

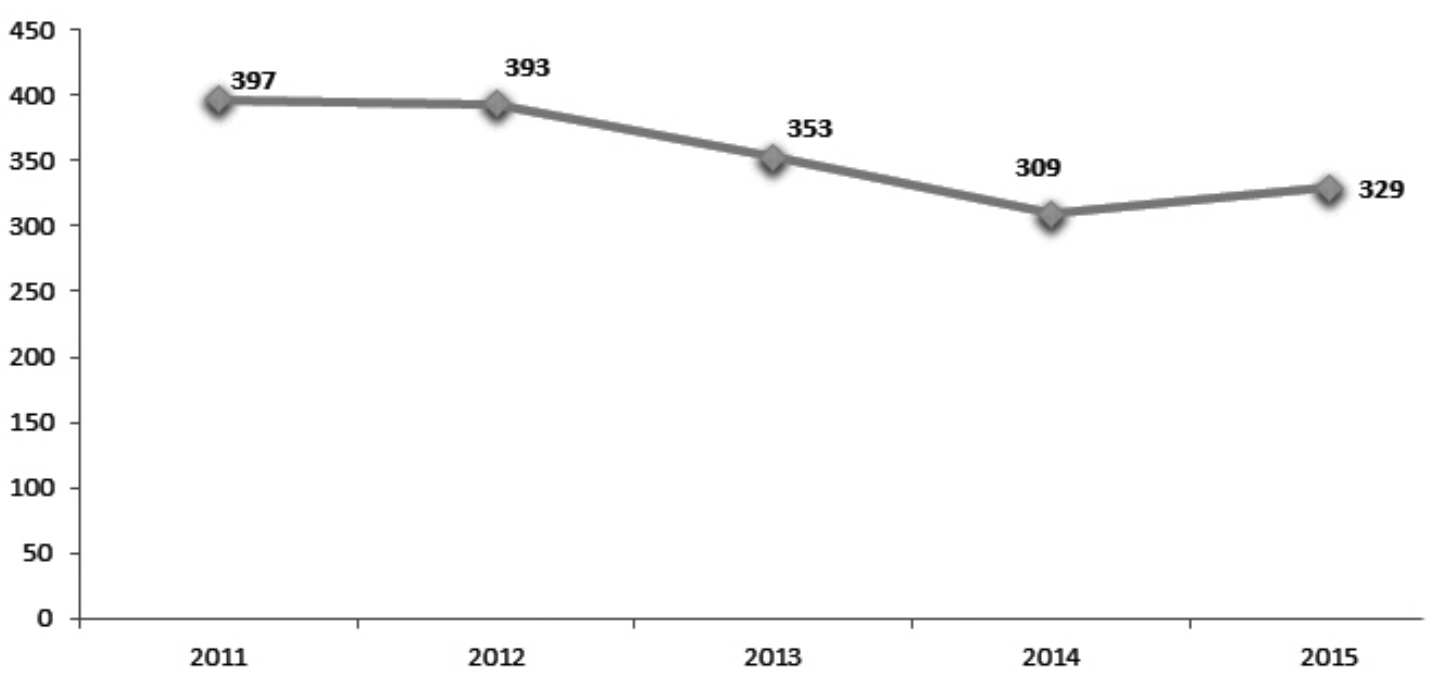

Fuente: Comité de Validación MCE - COISBOL.

Gráfica 3: COMPORTAMIENTO DE MUERTES POR CAUSAS EXTERNAS POR VARIABLES. ENERO - DICIEMBRE COMPARATIVO 2011 - 2015

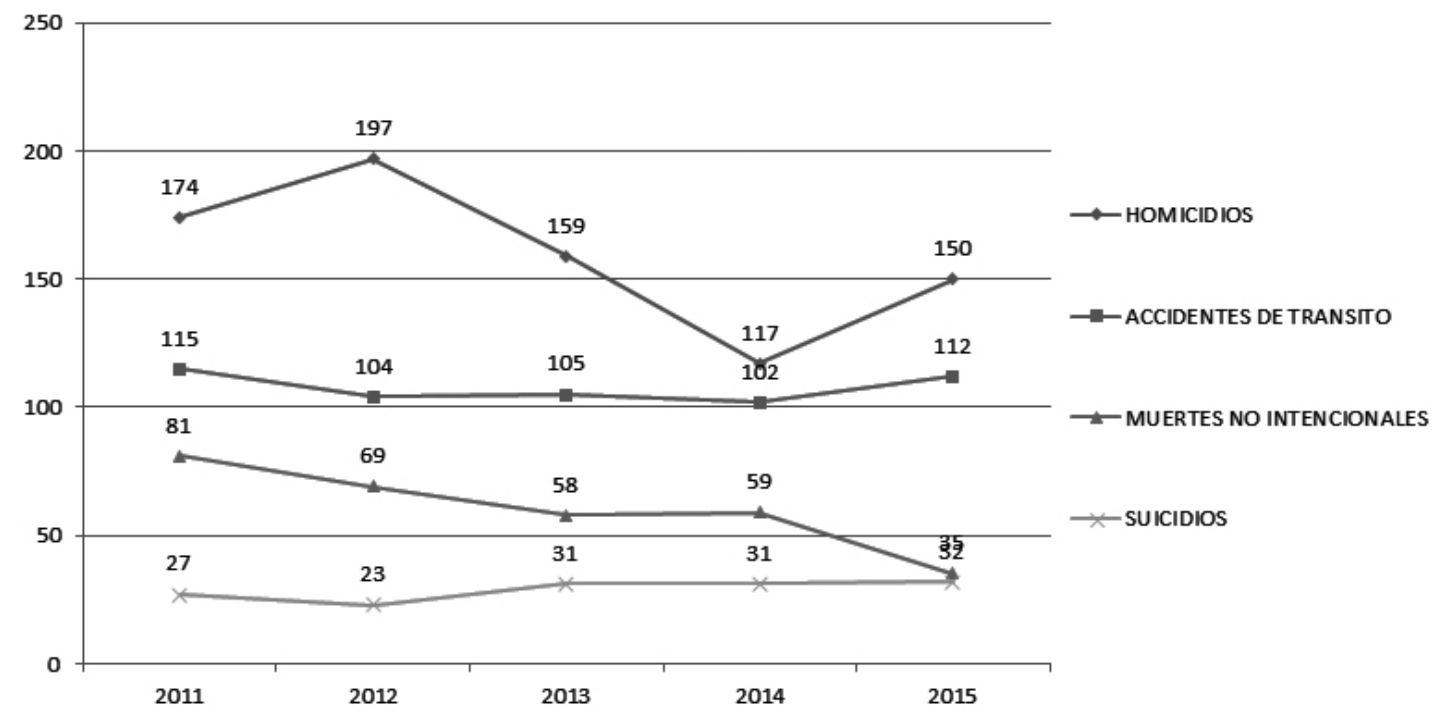

Fuente: Comité de Validación MCE - COISBOL. 
Es preciso mencionar, para destacar, que según el DANE la proyección de población estimada para Bolívar en 2015 (excluyendo Cartagena y sus corregimientos) es de 1.095.406 habitantes, lo que indica que la tasa de mortalidad por causa externa ha sido solo del 30,03\% para Bolívar ${ }^{6}$ Para tener un comparativo de referencia, según el informe publicado por Medicina Legal titulado Forensis 2014. Datos para la vida. Herramienta para la interpretación, intervención y prevención de lesiones de causa externa en Colombia ${ }^{7}$, durante el año 2014, esta institución recibió 25.225 casos por muertes violentas con una tasa de 52,92 casos por cada 100.000 habitantes, muy por encima de la misma tasa arriba señalada para el Departamento de Bolívar de 36,95 para el mismo año.

En 2015, en esos doce meses de Gobierno, la tasa de mortalidad por causa externa se ubica según los datos analizados en 30,03 para Bolívar -excluyendo a Cartagena que presenta una tasa de 52,95 incluido sus corregimientos-.

\section{PARA DESTACAR:}

- Enero - diciembre del 2015 ha sido el segundo periodo más seguro tomando como referencia las mediciones del 2011 a 2015, con 329 casos de MCE inferior al promedio de 356 casos del mismo periodo comparado.

- Los 150 casos de muerte por homicidio en Bolívar durante enero-diciembre del 2015 siguen estando por debajo del promedio del periodo comparado 2011-2015, que es de 159,4 casos promedio.

- Los accidentes de tránsito, segunda causa de muerte violenta en Bolívar durante este periodo, con 112 casos, se incrementaron respecto al año inmediatamente anterior.

- El número de muertes no intencionales disminuyó favorablemente. De los 59 casos del 2014 a solo 35 en 2015 (disminuyó del 2014 a 2015 en un 39,52\%)

- La tasa de mortalidad por causa externa enero-diciembre del 2015 ha sido solo del 30,03, muy por debajo del promedio nacional, tomando como referencia el último informe disponible publicado por Medicina Legal sobre "FORENSIS 2014 DATOS PARA LA VIDA" que presenta una tasa nacional de mortalidad por causa externa del 52,92 anual 2014, y tasa de 36,95 anual 2014 solo para Bolívar y 52,95 para Cartagena y sus corregimientos.

- Total MCE en 2015: 329

- 45,59\% Homicidios

-34,04\% Accidentes de Tránsito

$-10,63 \%$ Muertes No Intencionales

$-9,72 \%$ Suicidios

\footnotetext{
${ }^{6}$ Metodología de cálculo: MLCExt=(No.total de defunciones estimadas por lesiones de causa externa en una población / No. total de población ) x 100.000 (Tasas calculadas con base en las proyecciones de población DANE 2005-2020).

7 Instituto Nacional de Medicina Legal y Ciencias Forenses. (2014). Forensis 2014. Datos para la vida. Herramienta para la interpretación, intervención y prevención de lesiones de causa externa en Colombia. Recuperado de : http://www.medicinalegal.gov. co/documents/88730/1656998/Forensis+Interactivo+2014.24-JULpdf.pdf/9085ad79-d2a9-4c0d-a17b-f845ab96534b
} 


\section{HOMICIDIO}

El homicidio en Bolívar como causa de muerte violenta sigue experimentado un descenso en el último periodo objeto de análisis, enero a diciembre 2011 - 2015. De todos los periodos analizados, el 2014 y 2015 son los que menos casos de homicidio presentan con 117 y 150 casos respectivamente en todo el departamento, muy por debajo de los años precedentes, consolidándose cada vez más el departamento de Bolívar como Territorio Seguro.

\section{Grafica 4: COMPORTAMIENTO TOTAL DE MUERTES POR HOMICIDIO} ENERO - DICIEMBRE COMPARATIVO 2011 - 2015

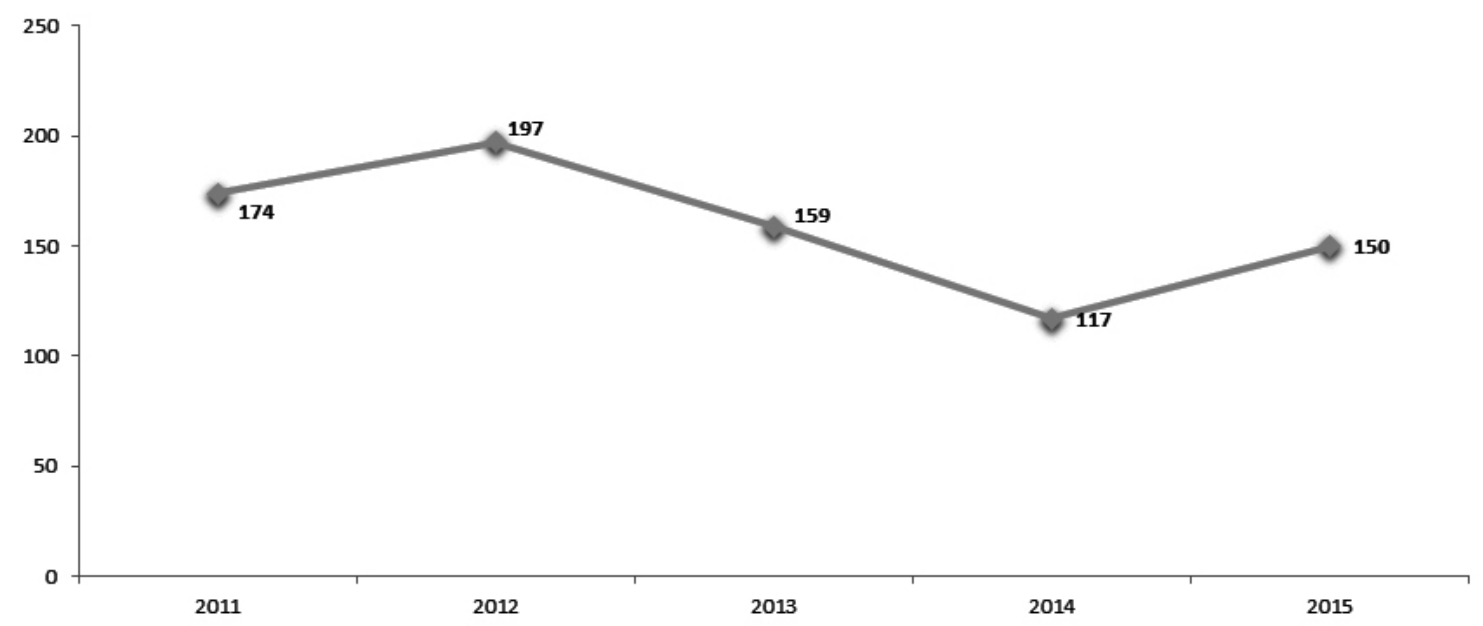

A nivel nacional, durante 2014 en Colombia, según cifras generadas por el Instituto Nacional de Medicina Legal y Ciencias Forenses, se registraron 12.626 homicidios, obteniéndose así una tasa de 26,49 por cada 100.000 habitantes, la más baja en los últimos diez años.

La tasa de mortalidad por homicidio durante el 2014 en el Departamento de Bolívar ha sido de 19,87 por cada 100.000 habitantes. En el 2015, esta Tasa se ubica en 13,69 por cada 100.000 habitantes, consolidándose el departamento de Bolívar como un territorio seguro en la defensa y preservación de la vida de sus habitantes.

El mes más violento en materia de homicidios de todos los periodos objeto de análisis fue julio, con 97 casos entre 2011 a 2015, disminuyendo paulatinamente en los meses sucesivos, agosto con 67 , septiembre con 72 , para incrementarse nuevamente en octubre con 79 casos. En el año, 2015, el mes más violento fue precisamente octubre con 25 casos, y los meses con mejores indicadores son junio con 4 casos y agosto con 5 , tal como se observa en la Tabla No. 01 que sigue: 
Gráfica 5: TOTAL DE MUERTES POR HOMICIDIOS

ENERO - DICIEMBRE 2011 - 2015

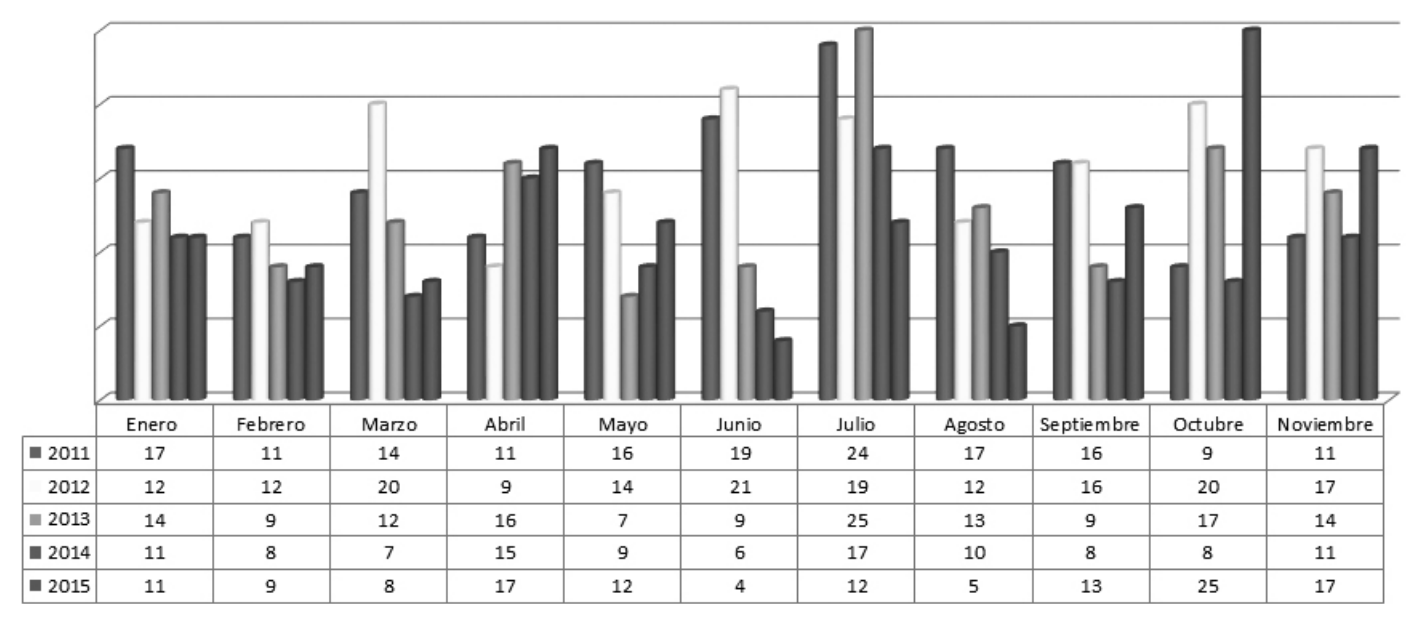

Fuente: Comité de Validación MCE - COISBOL.

TABLA 1: CANTIDAD DE HOMICIDIOS POR MES / AÑO ENERO - DICIEMBRE

\begin{tabular}{|c|c|c|c|c|c|c|}
\hline \multirow{2}{*}{ Meses } & \multicolumn{5}{|c|}{ Cantidad de Homicidios Por Año } & \multirow[b]{2}{*}{ TOTAL } \\
\hline & 2011 & 2012 & 2013 & 2014 & 2015 & \\
\hline Enero & 17 & 12 & 14 & 11 & 11 & 65 \\
\hline Febrero & 11 & 12 & 9 & 8 & 9 & 49 \\
\hline Marzo & 14 & 20 & 12 & 7 & 8 & 61 \\
\hline Abril & 11 & 9 & 16 & 15 & 17 & 68 \\
\hline Mayo & 16 & 14 & 7 & 9 & 12 & 58 \\
\hline Junio & 19 & 21 & 9 & 6 & 4 & 59 \\
\hline Julio & 24 & 19 & 25 & 17 & 12 & 97 \\
\hline Agosto & 17 & 12 & 13 & 10 & 5 & 57 \\
\hline Septiembre & 16 & 16 & 9 & 8 & 13 & 62 \\
\hline Octubre & 9 & 20 & 17 & 8 & 25 & 79 \\
\hline Noviembre & 11 & 17 & 14 & 11 & 17 & 64 \\
\hline Diciembre & 9 & 25 & 14 & 7 & 17 & 72 \\
\hline Total & 174 & 197 & 159 & 117 & 150 & \\
\hline $\begin{array}{l}\text { TASA DE HOMICIDOS POR CADA } \\
100.000 \text { HABITANTES EN BOLIIVAR } \\
\text { (EXCLUYENDO NEUTRALIZADOS) }\end{array}$ & 16,62 & 18,61 & 14,85 & 10,81 & 13,69 & \\
\hline
\end{tabular}

Fuente: Comité de Validación MCE - COISBOL.

Comparados los seis Zodes en que se subdivide el Departamento de Bolívar ${ }^{8}$, se observa que durante el periodo objeto de análisis 2011-2015, el Zodes Dique, presenta el mayor número de casos históricamente con un total de 262, seguidos por Magdalena

${ }^{8}$ Dado su extensión y las características sociales y económicas, la Asamblea Departamental organizó territorialmente a Bolívar desde el 2001 en seis (6) Zonas de Desarrollo Económico y Social, ZODES. 
Medio con 181 y luego La Mojana con 167. Vale anotar que para 2015, en el caso del Zodes Dique se presenta una mejoría frente al año anterior (2014) pasando de 44 a 36 casos, y es el periodo con menos número de homicidios desde el 2011 a corte enero diciembre. Destacable igual la reducción de los casos de homicidio en los Zodes Mojana y Momposina. Por el contrario, se observa que Zodes que habían logrado a 2014 una significativa reducción de los casos de homicidios, para 2015 presentan incrementos que requieren especial atención de la fuerza pública, como es el caso de Zodes Montes de María y Zodes Loba.

\section{Gráfico 6: COMPARATIVO MUERTE POR HOMICIDIO ENERO - DICIEMBRE 2011-2015 POR ZODES}

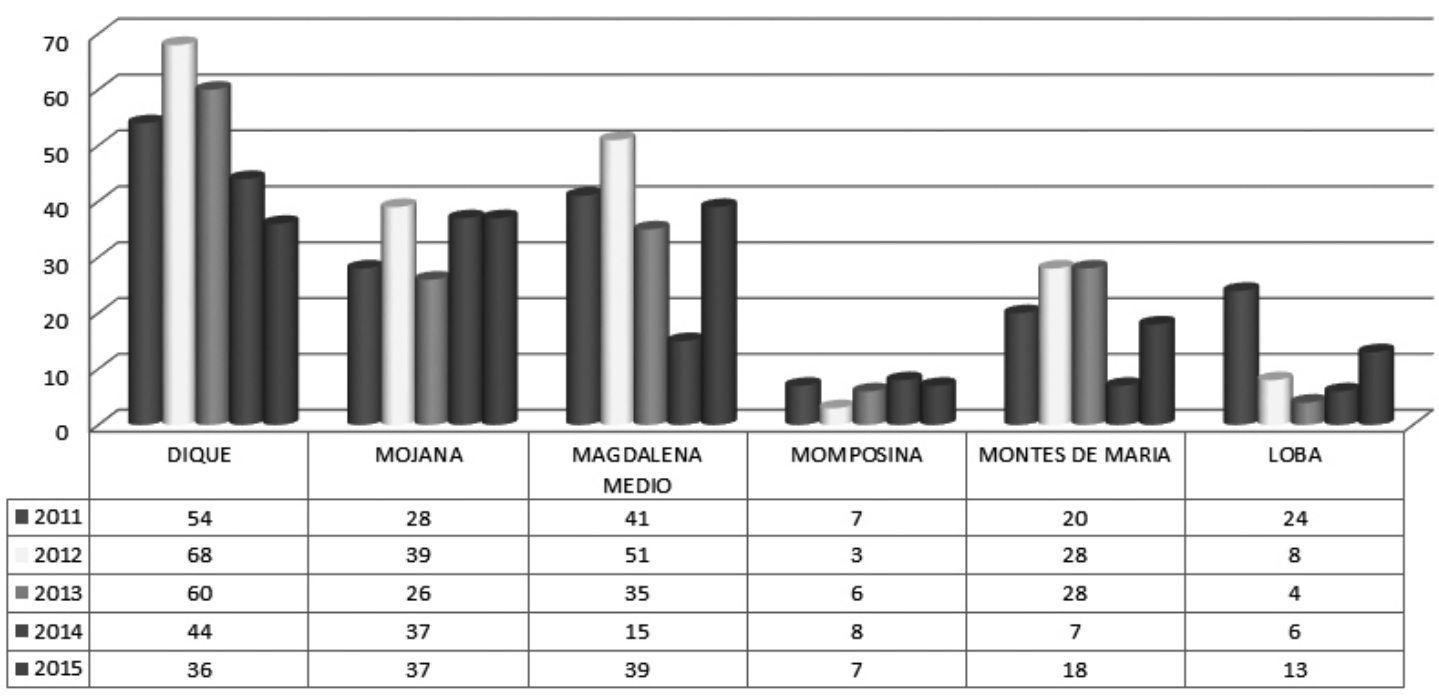

Fuente: Comité de Validación MCE - COISBOL.

Resulta notorio el incremento de las muertes violentas por homicidio en Regidor, pues de cero (0) casos presentados en el periodo enero - diciembre de los años 2011 a 2014, se pasó a cuatro (4) casos en el 2015. Otros municipios presentan aún presencia del ELN, y otras bandas delincuenciales y de crimen organizado, asociado a la extorsión y a la minería ilegal, lo que altera el orden público en la zona. Casos San Pablo, Morales, y Montecristo, entre otros. Al observar por municipios, se detalla que Altos del Rosario, Arroyo Hondo, Hatillo de Loba, San Cristóbal, San Fernando y Soplaviento, mantienen desde el 2012 cero (0) casos de homicidios, constituyéndose municipios seguros:

Tabla 2: COMPARATIVO MUERTE POR HOMICIDIO ENERO-DICIEMBRE 2011-2015 DISTRIBUIDO POR MUNICIPIOS

\begin{tabular}{|l|c|c|c|c|c|}
\hline \multicolumn{1}{|c|}{ MUNICIPIO } & $\mathbf{2 0 1 1}$ & $\mathbf{2 0 1 2}$ & $\mathbf{2 0 1 3}$ & $\mathbf{2 0 1 4}$ & $\mathbf{2 0 1 5}$ \\
\hline ACHI & 1 & 7 & 1 & 4 & 3 \\
\hline ALTOS DEL ROSARIO & 2 & 0 & 0 & 0 & 0 \\
\hline ARENAL & 2 & 3 & 1 & 1 & 5 \\
\hline ARJONA & 14 & 13 & 17 & 13 & 10 \\
\hline
\end{tabular}




\begin{tabular}{|c|c|c|c|c|c|}
\hline ARROYO HONDO & 1 & 0 & 1 & 0 & 1 \\
\hline BARRANCO DE LOBA & 7 & 0 & 1 & 2 & 0 \\
\hline CALAMAR & 1 & 0 & 0 & 4 & 1 \\
\hline CANTAGALLO & 2 & 2 & 5 & 1 & 1 \\
\hline CICUCO & 2 & 1 & 2 & 3 & 0 \\
\hline CLEMENCIA & 4 & 0 & 1 & 1 & 1 \\
\hline CORDOBA & 3 & 0 & 0 & 1 & 1 \\
\hline EL CARMEN DE BOLIVAR & 5 & 8 & 8 & 3 & 3 \\
\hline EL GUAMO & 0 & 0 & 0 & 1 & 0 \\
\hline EL PEÑON & 0 & 0 & 1 & 0 & 0 \\
\hline HATILLO DE LOBA & 0 & 0 & 0 & 0 & 1 \\
\hline MAGANGUE & 13 & 21 & 6 & 18 & 14 \\
\hline MAHATES & 2 & 4 & 2 & 2 & 4 \\
\hline MARGARITA & 0 & 0 & 0 & 1 & 3 \\
\hline MARIA LA BAJA & 10 & 10 & 14 & 2 & 6 \\
\hline MOMPOX & 5 & 2 & 4 & 2 & 2 \\
\hline MONTECRISTO & 5 & 6 & 6 & 3 & 8 \\
\hline MORALES & 1 & 2 & 1 & 4 & 10 \\
\hline NOROSI & 4 & 2 & 1 & 2 & 5 \\
\hline PINILLOS & 2 & 1 & 2 & 0 & 4 \\
\hline REGIDOR & 1 & 0 & 0 & 0 & 4 \\
\hline RIO VIEJO & 6 & 2 & 0 & 0 & 1 \\
\hline SAN CRISTOBAL & 0 & 0 & 0 & 0 & 0 \\
\hline SAN ESTANISLAO DE KOSTKA & 0 & 2 & 1 & 0 & 1 \\
\hline SAN FERNANDO & 0 & 0 & 0 & 0 & 0 \\
\hline SAN JACINTO & 1 & 7 & 3 & 0 & 1 \\
\hline SAN JACINTO DEL CAUCA & 2 & 0 & 1 & 0 & 1 \\
\hline SAN JUAN NEPOMUCENO & 0 & 2 & 3 & 0 & 5 \\
\hline SAN MARTIN DE LOBA & 4 & 4 & 1 & 2 & 3 \\
\hline SAN PABLO & 25 & 24 & 7 & 7 & 14 \\
\hline SANTA CATALINA & 0 & 1 & 1 & 1 & 0 \\
\hline SANTAROSA DE LIMA & 4 & 1 & 4 & 1 & 2 \\
\hline SANTA ROSA DEL SUR & 10 & 16 & 13 & 1 & 5 \\
\hline SIMITÍ & 1 & 4 & 8 & 1 & 4 \\
\hline SOPLAVIENTO & 0 & 0 & 0 & 0 & 0 \\
\hline TALAIGUA NUEVO & 0 & 0 & 0 & 2 & 1 \\
\hline TIQUISIO & 5 & 4 & 10 & 12 & 7 \\
\hline TURBACO & 17 & 34 & 29 & 16 & 14 \\
\hline TURBANA & 6 & 4 & 2 & 5 & 1 \\
\hline VILLANUEVA & 5 & 9 & 2 & 1 & 1 \\
\hline ZAMBRANO & 1 & 1 & 0 & 0 & 2 \\
\hline TOTAL & 174 & 197 & 159 & 117 & 150 \\
\hline
\end{tabular}

Fuente: Comité de Validación MCE - COISBOL. 
Por otro lado, para 2015, municipios como Arjona, Magangué, Morales, Tiquisio, Turbaco, San Pablo y Montecristo ya citados, presentan el mayor número de casos de muerte por causas externas por homicidios, aunque la mayoría hayan empezado a disminuir la tendencia histórica que venían presentado desde el año 2011.

\section{PARA DESTACAR:}

- El homicidio en Bolívar como causa de muerte violenta ha experimentado un descenso en el último periodo objeto de análisis, enero a diciembre 2011 - 2015, aunque en 2015 presentó un comportamiento irregular dado en un incremento frente al año anterior.

- El periodo enero - diciembre del 2015 presenta 150 casos de homicidio, rompiéndose la tendencia a disminuir que se venía consolidando entre 2011 (174 casos), 2012 (197 casos, siendo este el periodo más violento), 2013 y 2014 (que disminuyen a 159 y 117 casos respectivamente).

- La tasa de mortalidad por homicidio durante enero a diciembre del 2015 en Bolívar ha sido de 13,69 por cada 100.000 habitantes: la segunda más baja desde el 2011.

- El Zodes Dique, Mojana y Magdalena Medio presentan los mayores índices de homicidio en todo el departamento.

- Resulta notorio el incremento de las muertes violentas por homicidio en Regidor, pues de cero (0) casos presentados en el periodo enero - diciembre de los años 2011 a 2014, se pasó a cuatro (4) casos en el 2015

- Municipios Seguros: Altos del Rosario, San Cristóbal, San Fernando y Soplaviento mantienen desde 2012 cero (0) casos de homicidios, constituyéndose municipios seguros.

- Arjona, Magangué, Morales, Tiquisio, Turbaco, San Pablo y Montecristo ya citados, presentan el mayor número de casos de muerte por causas externas por homicidios, aunque la mayoría hayan empezado a disminuir la tendencia histórica que venían presentado desde el año 2011.

\section{ACCIDENTES DE TRANSITO}

En Bolívar, las muertes por accidentes de tránsito hasta el año 2014 estaban asociadas mayoritariamente a transporte en motocicletas. Es así como se observó que las muertes en accidente por motocicleta durante el 2011 al 2015 corresponden al 47,79\% (Datos de COISBOL). El resto de casos está asociado a causas por exceso de velocidad, imprudencia en la vía tanto de conductores, peatones o ciclistas, conductores que presuntamente por agotamiento se quedan dormidos frente al volante, aumento del tráfico vial en épocas de temporada alta de turismo, inobservancia de normas de seguridad en transporte fluvial, entre otros. Estas últimas causas precisamente son las que han significado un incremento en las MCE en el periodo enero - diciembre del 2015, ubicándola como la segunda causa de muertes por causas externas en el Departamento de Bolívar. En total 
112 casos presentados (2015) ubican este periodo por encima del promedio $(107,6)$ de los últimos cinco años comparados.

\section{Gráfica 7: COMPORTAMIENTO TOTAL DE MUERTES POR ACCIDENTES DE TRANSITO}

ENERO - DICIEMBRE $2011-2015$

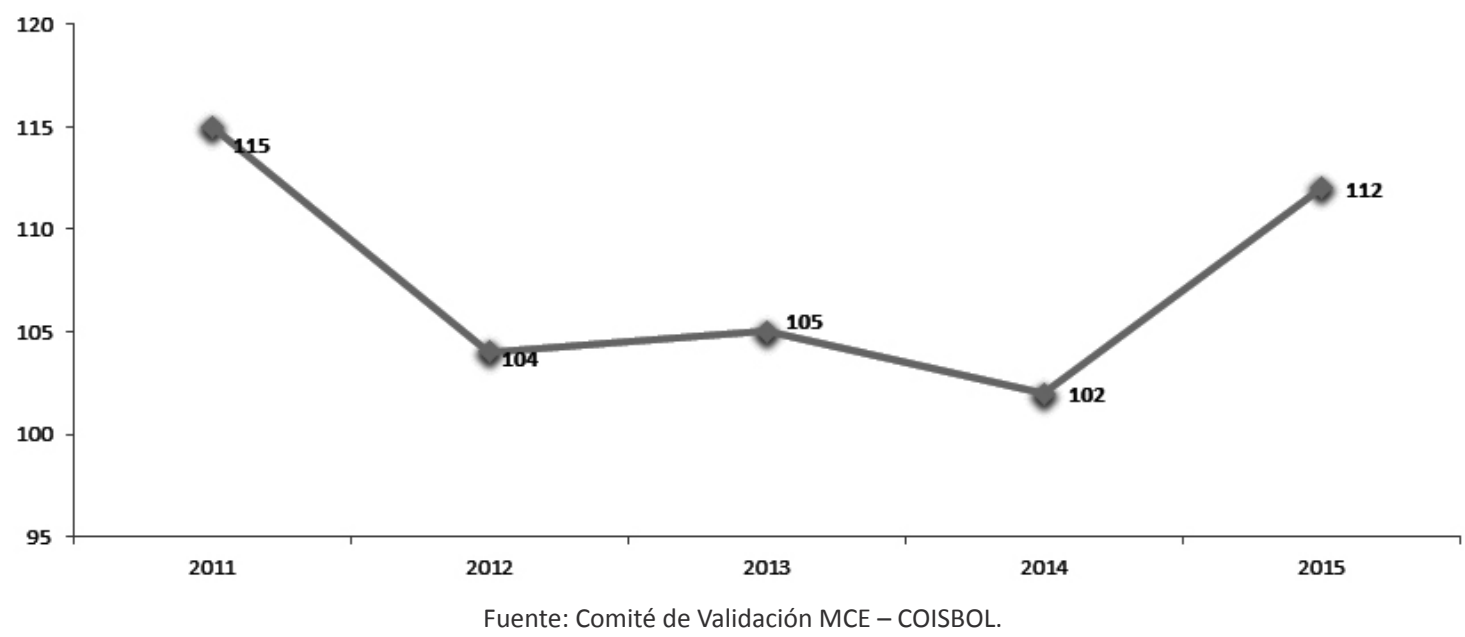

Diciembre y enero del 2015 son meses en los que tradicionalmente se incrementa el flujo de tráfico por las vías de Bolívar, especialmente en la Troncal de Occidente y la variante San Onofre, que conectan el interior del país con Cartagena, destino turístico tradicional, así como otros municipios del Centro de Bolívar. Igual análisis frente a los 12 casos presentados en el mes de marzo y 13 del mes de abril, en temporada turística asociada a la Semana Santa, que, igualmente incrementan el flujo de vehículos en las vías intermunicipales y departamentales.

\section{GRÁFICO 8: COMPARATIVO MUERTE POR ACCIDENTES DE TRÁNSITO ENERO - DICIEMBRE 2011-2015 POR MES}

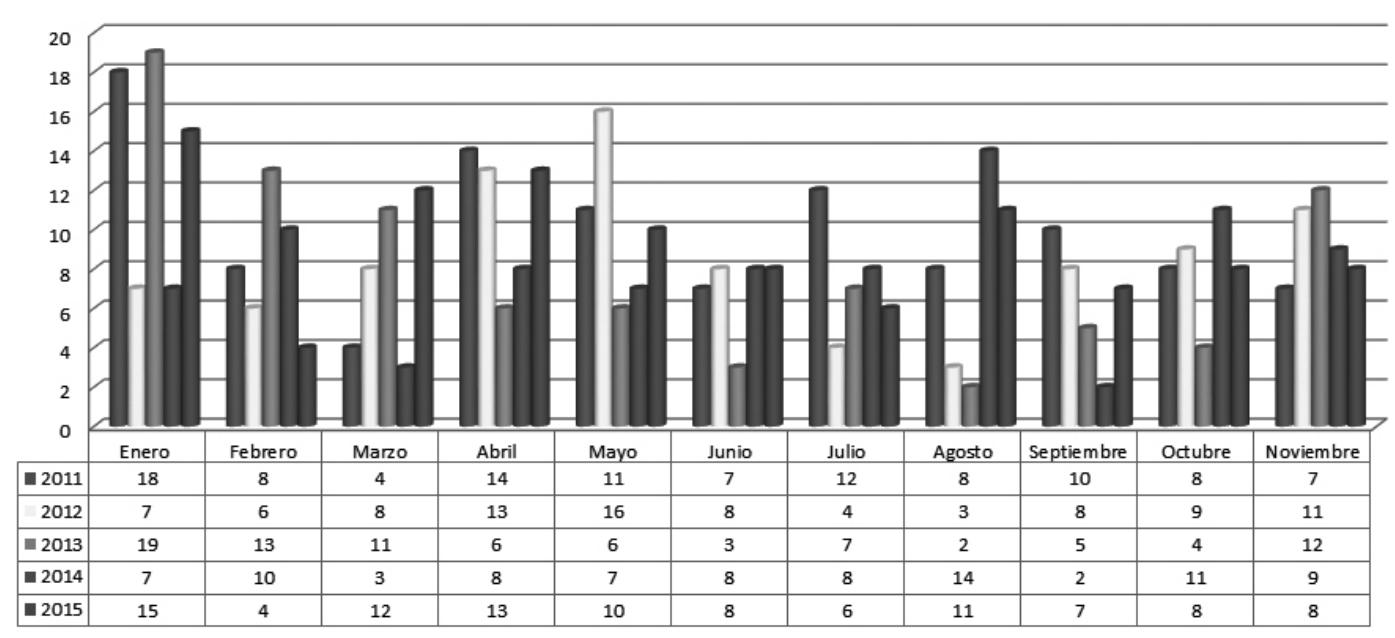

Fuente: Comité de Validación MCE - COISBOL. 
Ello explica que en el periodo 2011-2015 analizado el mayor número de casos estén concentrados en diciembre (61 casos), enero (66 casos) y abril (54 casos), tanto en vehículos particulares, como en servicio público de pasajeros, sin obviar la alta accidentalidad fatal que generan las motocicletas.

En términos de tasas por 100.000 habitantes, la de Colombia para el año 2014 es de 13,4 muertes (Instituto Nacional de Medicina Legal y Ciencias Forenses, 2014). La tasa de mortalidad por accidentes de tránsito durante el 2015 a corte diciembre en Bolívar fue de 10,22 por cada 100.000 habitantes, similar a los años precedentes (2014 con 9,42 y 2013 con 9,81). En el año 2014, para Cartagena, la Tasa fue de 12,22.

A pesar de estar por debajo de la tasa nacional, la accidentalidad sigue siendo una de las tareas sobre la que autoridades civiles municipales y fuerza pública deben priorizar acciones y planes para preservar la vida. La Troncal de Occidente atraviesa los Montes de María y los municipios de Arjona y Bolívar en el norte del Departamento. Ello explica que sean los Zodes Montes de María, y Dique, los de mayor ocurrencia de accidentes fatales, como se observa en la gráfica siguiente:

GRÁFICO 9: COMPARATIVO MUERTE POR ACCIDENTES DE TRÁNSITO POR ZODES ENERO - DICIEMBRE 2011-2015

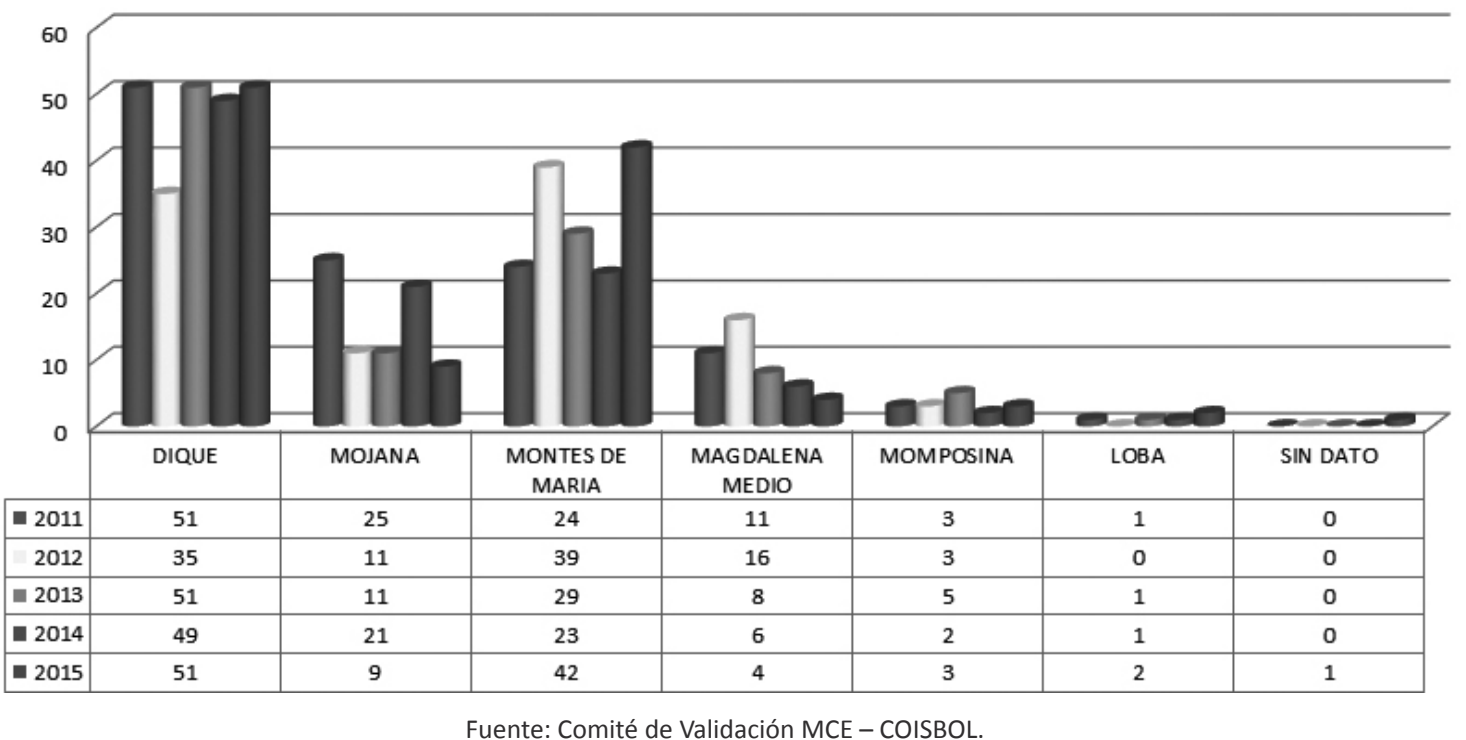

El comportamiento de esta variable analizada por municipios, ubica a El Carmen de Bolívar y Turbaco con los mayores índices de accidentalidad fatal, seguidos por el Municipio de Arjona, Magangué, y San Juan Nepomuceno, todos asociados a un constante y alto flujo de circulación de motocicletas, que sumado a la imprudencia en las vías, generó una alta mortalidad. 
Tabla 3: COMPARATIVO MUERTE POR ACCIDENTE DE TRANSITO ENERO-DICIEMBRE 2011-2015 DISTRIBUIDO POR MUNICIPIOS

\begin{tabular}{|c|c|c|c|c|c|}
\hline MUNICIPIO & 2011 & 2012 & 2013 & 2014 & 2015 \\
\hline $\mathrm{ACHI}$ & 0 & 0 & 0 & 0 & 0 \\
\hline ALTOS DEL ROSARIO & 0 & 0 & 0 & 1 & 0 \\
\hline ARENAL & 1 & 1 & 0 & 1 & 0 \\
\hline ARJONA & 17 & 14 & 17 & 12 & 12 \\
\hline ARROYO HONDO & 0 & 2 & 0 & 0 & 0 \\
\hline BARRANCO DE LOBA & 0 & 0 & 1 & 0 & 0 \\
\hline CALAMAR & 3 & 1 & 3 & 1 & 3 \\
\hline CANTAGALLO & 0 & 2 & 0 & 0 & 0 \\
\hline CICUCO & 0 & 0 & 0 & 0 & 0 \\
\hline CLEMENCIA & 3 & 0 & 1 & 1 & 4 \\
\hline CORDOBA & 1 & 0 & 1 & 1 & 2 \\
\hline EL CARMEN DE BOLIVAR & 9 & 11 & 6 & 4 & 19 \\
\hline EL GUAMO & 0 & 0 & 2 & 0 & 0 \\
\hline EL PEÑON & 0 & 0 & 0 & 0 & 0 \\
\hline HATILLO DE LOBA & 0 & 0 & 0 & 0 & 0 \\
\hline MAGANGUE & 16 & 11 & 10 & 14 & 7 \\
\hline MAHATES & 8 & 3 & 8 & 1 & 5 \\
\hline MARGARITA & 1 & 0 & 0 & 1 & 0 \\
\hline MARIA LA BAJA & 2 & 5 & 6 & 8 & 6 \\
\hline MOMPOX & 2 & 2 & 4 & 0 & 3 \\
\hline MONTECRISTO & 0 & 0 & 0 & 0 & 1 \\
\hline MORALES & 1 & 1 & 0 & 0 & 0 \\
\hline NOROSI & 0 & 0 & 0 & 0 & 0 \\
\hline PINILLOS & 9 & 0 & 0 & 4 & 0 \\
\hline REGIDOR & 0 & 0 & 0 & 0 & 0 \\
\hline RIO VIEJO & 1 & 0 & 0 & 0 & 1 \\
\hline SAN CRISTOBAL & 0 & 0 & 0 & 0 & 0 \\
\hline SAN ESTANISLAO DE KOSTKA & 1 & 0 & 0 & 0 & 1 \\
\hline SAN FERNANDO & 0 & 0 & 0 & 0 & 0 \\
\hline SAN JACINTO & 2 & 1 & 1 & 6 & 3 \\
\hline SAN JACINTO DEL CAUCA & 0 & 0 & 0 & 0 & 1 \\
\hline SAN JUAN DE NEPOMUCENO & 6 & 22 & 9 & 4 & 9 \\
\hline SAN MARTIN DE LOBA & 0 & 0 & 0 & 0 & 1 \\
\hline SAN PABLO & 3 & 6 & 1 & 2 & 1 \\
\hline SANTA CATALINA & 2 & 6 & 2 & 0 & 2 \\
\hline SANTA ROSA DEL SUR & 4 & 5 & 6 & 1 & 2 \\
\hline SANTA ROSA DE LIMA & 0 & 2 & 0 & 1 & 1 \\
\hline SIMITI & 2 & 1 & 1 & 2 & 1 \\
\hline SOPLAVIENTO & 0 & 0 & 0 & 0 & 0 \\
\hline
\end{tabular}




\begin{tabular}{|l|c|c|c|c|c|}
\hline TALAIGUA NUEVO & 0 & 1 & 1 & 1 & 0 \\
\hline TIQUISIO & 0 & 0 & 1 & 3 & 1 \\
\hline TURBACO & 14 & 3 & 17 & 20 & 17 \\
\hline TURBANA & 3 & 1 & 2 & 13 & 5 \\
\hline VILLANUEVA & 0 & 3 & 1 & 0 & 1 \\
\hline ZAMBRANO & 4 & 0 & 4 & 0 & 2 \\
\hline SIN DATO & 0 & 0 & 0 & 0 & 1 \\
\hline TOTAL & 115 & 104 & 105 & 102 & 112 \\
\hline
\end{tabular}

Fuente: Comité de Validación MCE - COISBOL

Por otro lado, se destacan Municipios con cero (0) fatalidad por accidentes de tránsito entre 2011 y 2015, como son: Achí, Cicuco, El Peñón, Hatillo de Loba, Norosí, Regidor, San Cristóbal, San Fernando, y Soplaviento.

\section{PARA DESTACAR:}

- Accidentes de Tránsito con resultados fatales han incrementado el número de MCE en el periodo enero - diciembre del 2015, ubicándola como la segunda causa de muertes por causas externas en el departamento de Bolívar.

- Enero y marzo-abril (Semana Santa) presentan el mayor número de casos asociados al incremento del flujo vehicular por razones de turismo, concentrados principalmente en la carretera Troncal de Occidente.

- Para lograr a futuro una reducción significativa es perentorio redoblar los controles y las campañas de prevención vial en la vía de mayor ocurrencia de siniestros como es la Troncal de Occidente.

- Tasa de Mortalidad enero-diciembre del 2015 por causa de accidentes de tránsito: 10,22, superando a la registrada en el 2014 que fue de 9,42 para Bolívar, aunque menor que la registrada para el Distrito de Cartagena que fue de 12,22 incluido sus corregimientos.

- El Carmen de Bolívar, además de Turbaco y Arjona (carretera Troncal) y Magangué son los municipios con mayor accidentalidad fatal en Bolívar.

\section{MUERTES NO INTENCIONALES}

Representan solo el 10,63\% del total de MCE del Departamento de Bolívar durante el periodo enero - diciembre 2015 , siendo la tercera causa de muertes violentas.

Esta variable de MCE no intencionales presenta a 2015 una reducción significativa frente a los años anteriores, 59,32\% respecto al 2014, con una tasa de mortalidad de 3,19 que la ubica como la mejor desde el 2011 (2011 tuvo una tasa de mortalidad de 7,74 con un descenso significativo ubicándose en 2014 en 5,45) ${ }^{9}$ A nivel nacional, en el año 2014 hubo una tasa de 5,68 casos por cada 100.000 habitantes.

\footnotetext{
${ }^{9}$ Según Medicina Legal, en 2014 la tasa fue de 4,39 para el Departamento de Bolívar, no obstante para este análisis se validaron los datos arrojando la tasa en 5,45 para Bolívar en el año 2014.
} 


\section{Gráfica 10: COMPORTAMIENTO TOTAL DE MUERTES NO INTENCIONALES}

ENERO - DICIEMBRE COMPARATIVO 2011 - 2015

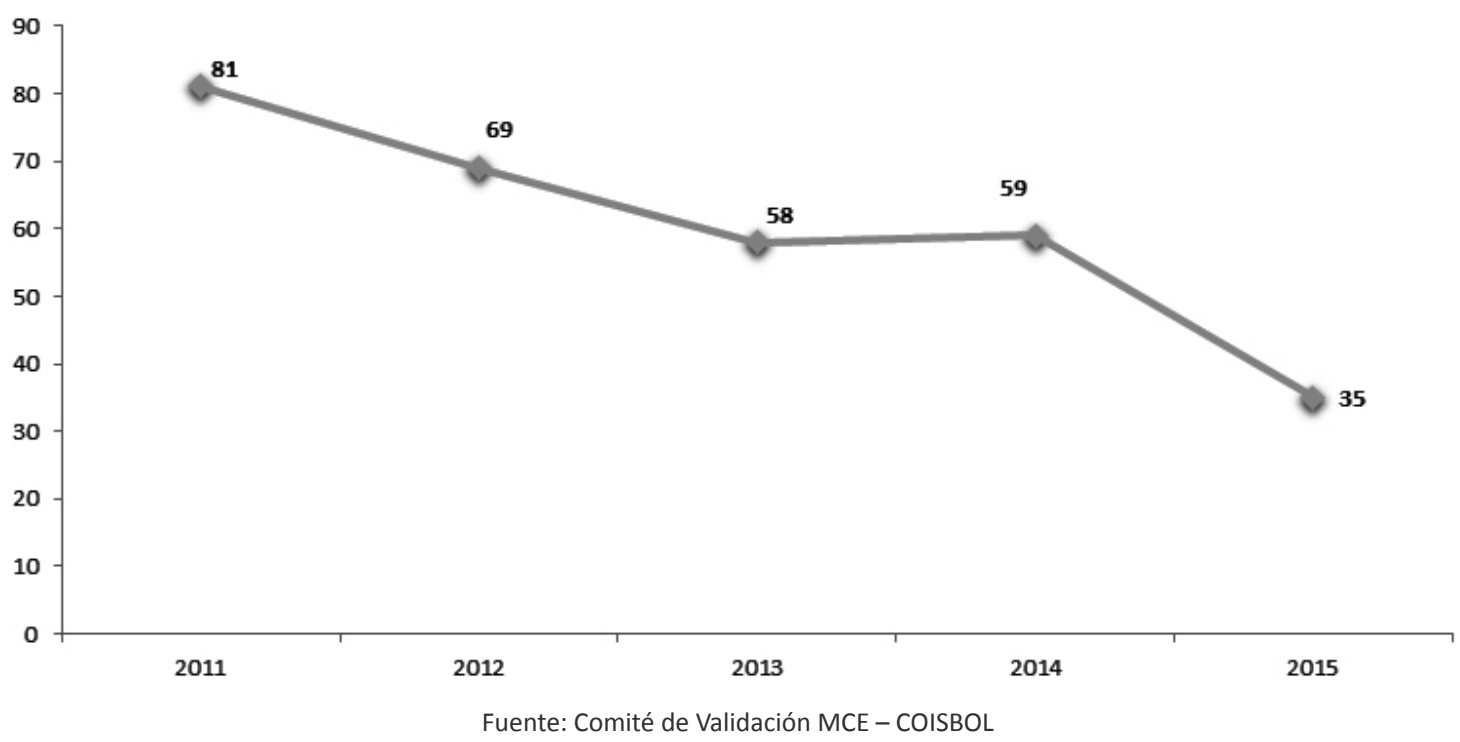

Los meses de mayor ocurrencia de muertes no intencionales han sido enero (39 casos acumulados en el periodo) y julio (34 casos acumulados). No obstante, el año 2015 presenta cifras generales muy por debajo de los años precedentes, que presentan tasas de muertes no intencionales mayores. El mes de Diciembre de 2015 cerró con cinco (5) casos, llegando a 22 casos total acumulados entre 2011-2015.

\section{Gráfico 11: COMPARATIVO MUERTE NO INTENCIONALES ENERO - DICIEMBRE 2011-2015 POR MES}

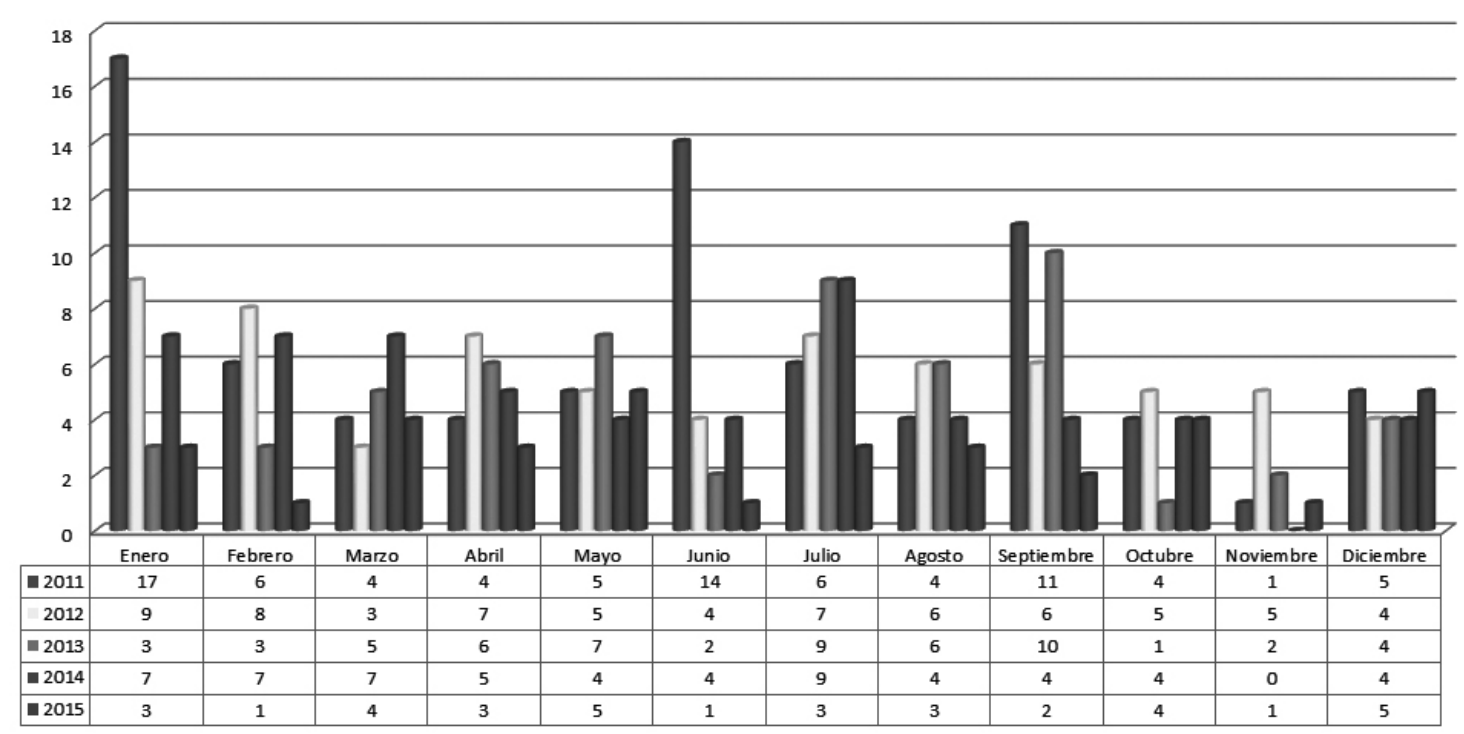

Fuente: Comité de Validación MCE - COISBOL 
Se observa que las muertes no intencionales han venido decreciendo desde el año 2011, con una sola excepción en mayo de 2015 donde se presentaron 5 casos superando a mayo del 2014 con 4 casos. El 2015, en general, ha sido el año con mejores resultados en Bolívar a partir de la medición de este indicador.La disminución de MCE no intencionales en 2015 se refleja en los indicadores de todos los Zodes, y a pesar de que la Mojana tiene el mayor número de casos en diciembre de 2015 en total, estos son 35,29\% menos que en el 2014 con 17 casos.

\section{GRÁFICO 12: COMPARATIVO MUERTE NO INTENCIONALES POR ZODES ENERO - DICIEMBRE 2011-2015}

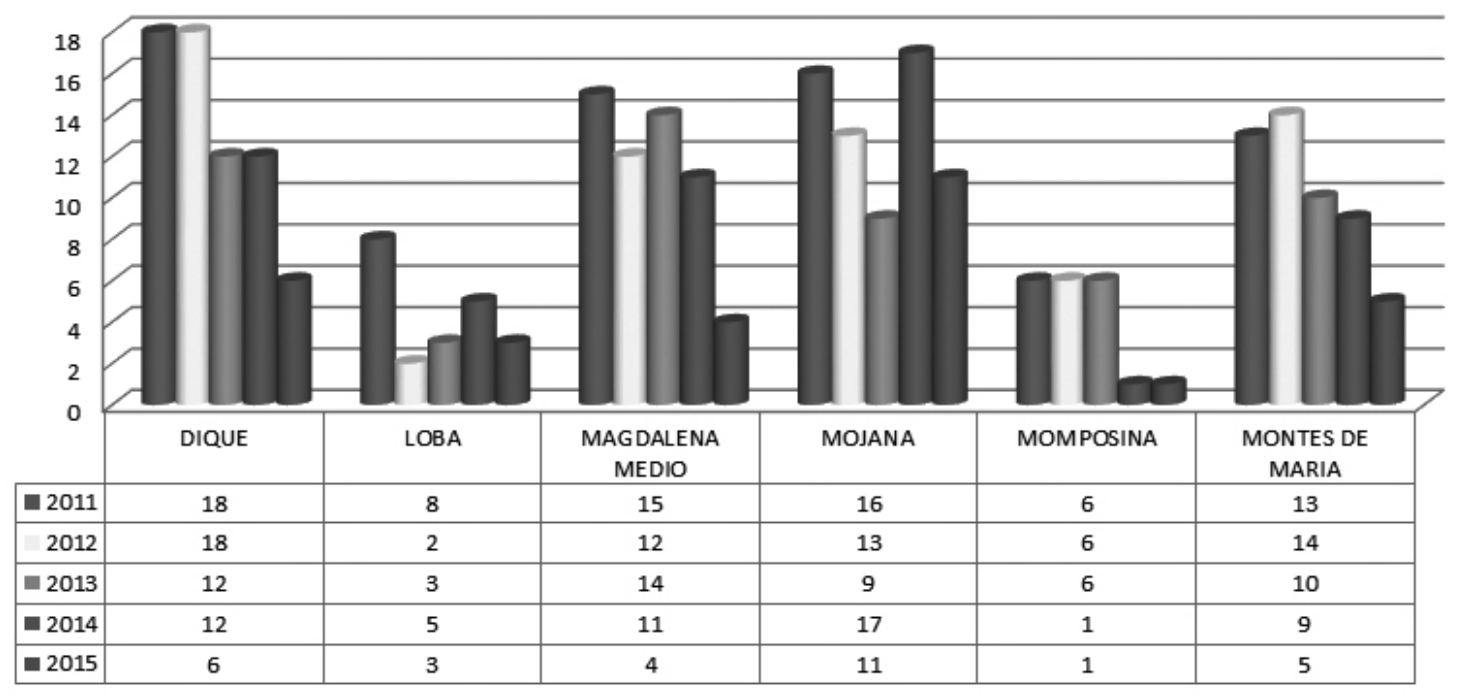

Fuente: Comité de Validación MCE - COISBOL

Es importante destacar que para lo corrido del año 2015 en Zodes Momposina solo se presentó un caso de Muerte No Intencional, igual que en 2014. Los municipios con mayores casos en esta variable son Magangué, Santa Rosa del Sur, Turbaco, Arjona y Marialabaja.

Tabla 4: COMPARATIVO MUERTE POR MUERTE NO INTENCIONAL ENERO-DICIEMBRE 2011-2015 DISTRIBUIDO POR MUNICIPIOS

\begin{tabular}{|l|c|c|c|c|c|}
\hline \multicolumn{1}{|c|}{ MUNICIPIO } & $\mathbf{2 0 1 1}$ & $\mathbf{2 0 1 2}$ & $\mathbf{2 0 1 3}$ & $\mathbf{2 0 1 4}$ & $\mathbf{2 0 1 5}$ \\
\hline ACHI & 0 & 2 & 0 & 1 & 0 \\
\hline ALTOS DEL ROSARIO & 1 & 0 & 0 & 0 & 0 \\
\hline ARENAL & 0 & 2 & 0 & 0 & 0 \\
\hline ARJONA & 1 & 4 & 6 & 3 & 5 \\
\hline ARROYO HONDO & 0 & 0 & 0 & 0 & 1 \\
\hline BARRANCO DE LOBA & 1 & 0 & 1 & 0 & 2 \\
\hline CALAMAR & 0 & 0 & 2 & 1 & 1 \\
\hline
\end{tabular}




\begin{tabular}{|c|c|c|c|c|c|}
\hline CANTAGALLO & 2 & 0 & 1 & 0 & 1 \\
\hline CICUCO & 1 & 1 & 1 & 0 & 0 \\
\hline CLEMENCIA & 2 & 0 & 0 & 2 & 0 \\
\hline CORDOBA & 1 & 0 & 1 & 1 & 0 \\
\hline EL CARMEN DE BOLIVAR & 4 & 1 & 4 & 3 & 1 \\
\hline EL GUAMO & 0 & 1 & 0 & 1 & 0 \\
\hline EL PEÑON & 1 & 0 & 0 & 1 & 0 \\
\hline HATILLO DE LOBA & 0 & 0 & 2 & 0 & 0 \\
\hline MAGANGUE & 14 & 8 & 7 & 10 & 6 \\
\hline MAHATES & 3 & 0 & 1 & 3 & 0 \\
\hline MARGARITA & 2 & 0 & 0 & 0 & 0 \\
\hline MARIA LA BAJA & 5 & 7 & 4 & 3 & 2 \\
\hline MOMPOX & 2 & 4 & 1 & 1 & 2 \\
\hline MONTECRISTO & 0 & 0 & 1 & 1 & 1 \\
\hline MORALES & 0 & 0 & 1 & 1 & 1 \\
\hline NOROSI & 0 & 0 & 0 & 0 & 1 \\
\hline PINILLOS & 1 & 1 & 1 & 3 & 3 \\
\hline REGIDOR & 2 & 0 & 0 & 2 & 0 \\
\hline RIO VIEJO & 1 & 1 & 2 & 1 & 0 \\
\hline SAN CRISTOBAL & 0 & 0 & 0 & 0 & 0 \\
\hline SAN ESTANISLAO DE KOSTKA & 0 & 0 & 0 & 0 & 0 \\
\hline SAN FERNANDO & 0 & 0 & 0 & 1 & 0 \\
\hline SAN JACINTO & 0 & 0 & 0 & 1 & 1 \\
\hline SAN JACINTO DEL CAUCA & 0 & 0 & 1 & 0 & 0 \\
\hline SAN JUAN NEPOMUCENO & 2 & 5 & 1 & 1 & 0 \\
\hline SAN MARTIN DE LOBA & 2 & 1 & 0 & 1 & 0 \\
\hline SAN PABLO & 3 & 4 & 7 & 3 & 0 \\
\hline SANTA CATALINA & 2 & 1 & 0 & 0 & 0 \\
\hline SANTA ROSA DE LIMA & 1 & 4 & 1 & 0 & 0 \\
\hline SANTA ROSA DEL SUR & 7 & 6 & 4 & 6 & 1 \\
\hline SIMITI & 4 & 2 & 3 & 1 & 1 \\
\hline SOPLAVIENTO & 0 & 0 & 0 & 1 & 0 \\
\hline TALAIGUA NUEVO & 1 & 2 & 2 & 0 & 0 \\
\hline TIQUISIO & 2 & 2 & 0 & 2 & 1 \\
\hline TURBACO & 6 & 9 & 2 & 2 & 3 \\
\hline TURBANA & 2 & 1 & 0 & 0 & 0 \\
\hline VILLANUEVA & 3 & 0 & 1 & 1 & 0 \\
\hline ZAMBRANO & 2 & 0 & 0 & 1 & 1 \\
\hline TOTAL & 81 & 69 & 58 & 59 & 35 \\
\hline
\end{tabular}

Fuente: Comité de Validación MCE - COISBOL 


\section{PARA DESTACAR:}

- Las MCE No Intencionales representan solo el 10,63\% del total de MCE del departamento de Bolívar durante el periodo enero - diciembre del 2015, siendo la tercera causa de muertes violentas con 35 casos en 2015.

- El 2015 ha sido el año más seguro en Bolívar a partir de la medición de este indicador.

- La tasa de mortalidad por cada 100.000 habitantes a diciembre del 2015 por esta causa es de solo 3,19 por cada cien mil habitantes. En el 2014 esta tasa se ubicó en 5,45, la más baja desde el 2011.

\section{SUICIDIOS}

Para el Instituto Nacional de Medicina Legal:

el suicidio es un problema de salud pública que ocupa un lugar importante entre las primeras diez causas de mortalidad en el mundo; esto ocurre independientemente de la geografía, religión, posición socioeconómica, etnia, cultura, entre otros. Las causas que inducen al suicidio son diversas, incluyen trastornos psiquiátricos y factores detonantes biológicos y psicosociales. El suicidio es una acción por la cual una persona acaba con su propia vida y está clasificado como conducta autodestructiva" (Zapata, et al., 2014, p.54).

Bajo esa premisa, el suicidio como causa de muerte es objeto de medición para tener insumos que permita desde las políticas públicas y acciones gubernamentales atender esta causa de mortalidad.

En Bolívar, los casos de suicidios son muy escasos y por ende llamativos ante la opinión pública y la ciudadanía. En el periodo objeto de análisis para 2015 solo se presentaron 32 casos, esto es el 9,72\% del total de MCE, siendo la tasa de mortalidad por esta causa equivalente a 2,92 (2015). La tendencia se mantiene similar al periodo 2011-2015, objeto de análisis.

GRÁFICA 13: COMPORTAMIENTO TOTAL DE MUERTES POR SUICIDIO ENERO A DICIEMBRE COMPARATIVO 2011 - 2015

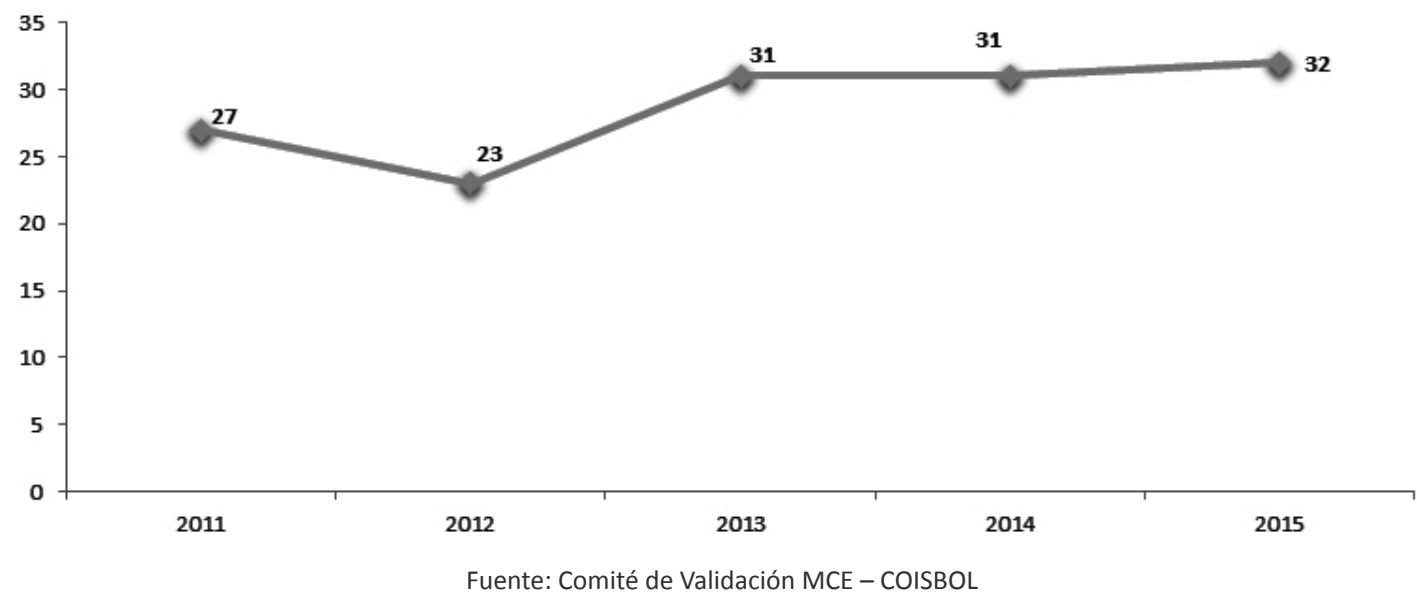


En el año 2014 el sistema médico legal colombiano reportó una tasa de mortalidad por suicidio de 4,33 casos por cada 100.000 habitantes a nivel nacional, similar a la reportada durante los últimos 9 años. Para el Departamento de Bolívar, la tasa de muertes por suicidio por casa 100.000 habitantes de 2,86 para 2014. En lo corrido del año (2015) esta tasa se ubica en 2,92 por cada 100.000 habitantes. El mayor número de casos se presenta históricamente en el mes de enero, abril, julio, y el último trimestre de cada año. Enero presuntamente asociado a una alteración en los estados emocionales posterior a las fiestas navideñas y de año nuevo.

GRÁFICO 14: COMPARATIVO MUERTE POR SUICIDIOS ENERO - DICIEMBRE 2011-2015 POR MES

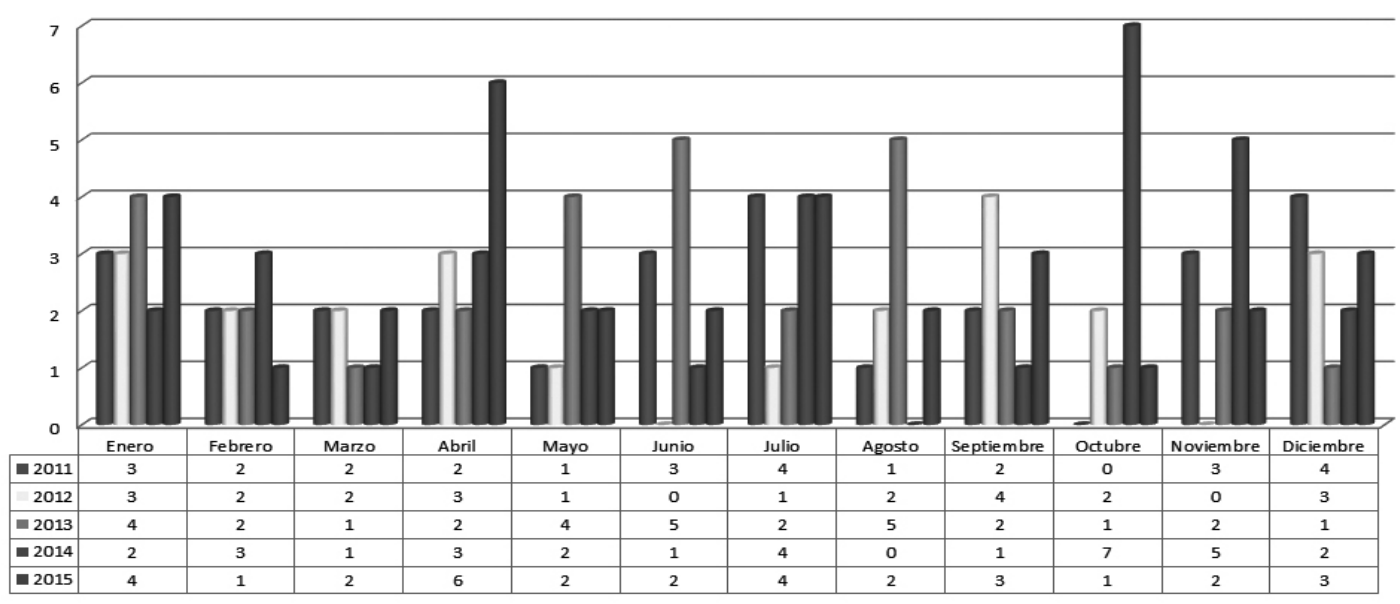

Fuente: Comité de Validación MCE - COISBOL

Aunque no sea significativo por el número tan reducido de casos es preciso anotar que los casos de suicidio están concentrados en los municipios que integran el Zodes Dique, y el Zodes Montes de María, seguidos por la Mojana.

GRÁFICO 15: COMPARATIVO MUERTE POR SUICIDOS ENERO - DICIEMBRE 2011-2015 POR ZODES

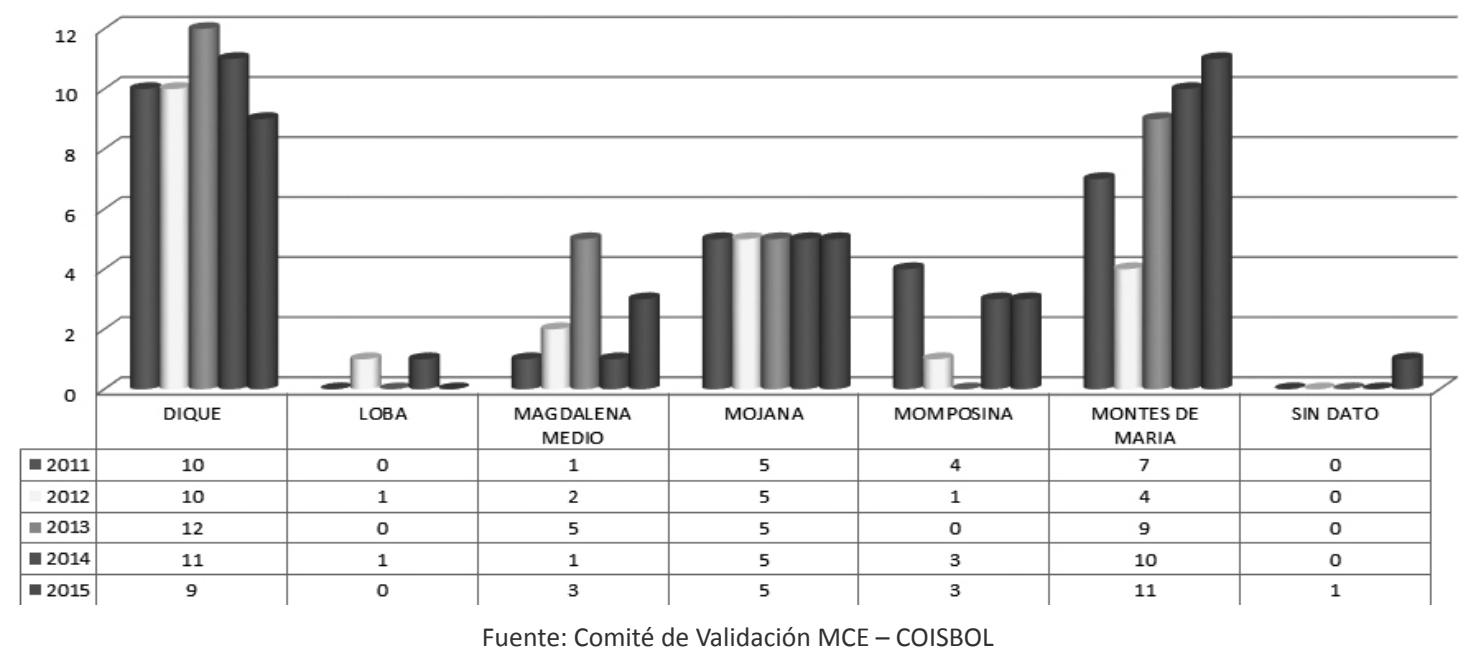


Son los grandes municipios de Bolívar, en términos de poblacionales, los que presentan los mayores casos de suicidios históricamente, encabezados por Magangué, seguido por Turbaco y El Carmen de Bolívar.

Tabla 5: COMPARATIVO MUERTE POR SUICIDIO DISTRIBUIDO POR MUNICIPIOS ENERO - DICIEMBRE 2011-2015

\begin{tabular}{|c|c|c|c|c|c|}
\hline MUNICIPIO & 2011 & 2012 & 2013 & 2014 & 2015 \\
\hline $\mathrm{ACHI}$ & 0 & 0 & 0 & 0 & 1 \\
\hline ALTOS DEL ROSARIO & 0 & 0 & 0 & 0 & 0 \\
\hline ARENAL & 1 & 0 & 0 & 0 & 0 \\
\hline ARJONA & 3 & 2 & 4 & 2 & 2 \\
\hline ARROYO HONDO & 0 & 0 & 0 & 0 & 0 \\
\hline BARRANCO DE LOBA & 0 & 0 & 0 & 1 & 0 \\
\hline CALAMAR & 1 & 1 & 2 & 0 & 0 \\
\hline CANTAGALLO & 0 & 0 & 1 & 0 & 1 \\
\hline CICUCO & 1 & 0 & 0 & 0 & 0 \\
\hline CLEMENCIA & 0 & 0 & 0 & 0 & 0 \\
\hline CORDOBA & 2 & 0 & 0 & 0 & 1 \\
\hline EL CARMEN DE BOLIVAR & 3 & 2 & 4 & 5 & 5 \\
\hline EL GUAMO & 0 & 0 & 1 & 0 & 0 \\
\hline EL PEÑON & 0 & 0 & 0 & 0 & 0 \\
\hline HATILLO DE LOBA & 0 & 0 & 0 & 0 & 0 \\
\hline MAGANGUE & 2 & 5 & 4 & 5 & 4 \\
\hline MAHATES & 2 & 1 & 1 & 1 & 1 \\
\hline MARIA LA BAJA & 1 & 0 & 1 & 2 & 3 \\
\hline MARGARITA & 2 & 0 & 0 & 0 & 0 \\
\hline MOMPOS & 0 & 1 & 0 & 3 & 1 \\
\hline MONTECRISTO & 0 & 0 & 0 & 0 & 0 \\
\hline MORALES & 0 & 0 & 0 & 1 & 0 \\
\hline NOROSI & 0 & 0 & 0 & 0 & 0 \\
\hline PINILLOS & 0 & 0 & 1 & 0 & 0 \\
\hline REGIDOR & 0 & 0 & 0 & 0 & 0 \\
\hline RIO VIEJO & 0 & 0 & 0 & 0 & 0 \\
\hline SAN CRISTOBAL & 0 & 1 & 0 & 0 & 0 \\
\hline SAN ESTANISLAO DE KOSTKA & 0 & 0 & 0 & 0 & 0 \\
\hline SAN FERNANDO & 1 & 0 & 0 & 0 & 1 \\
\hline SAN JACINTO & 1 & 1 & 1 & 1 & 0 \\
\hline SAN JACINTO DEL CAUCA & 0 & 0 & 0 & 0 & 0 \\
\hline SAN JUAN NEPOMUCENO & 0 & 1 & 2 & 2 & 2 \\
\hline SAN MARTIN DE LOBA & 0 & 1 & 0 & 0 & 0 \\
\hline SAN PABLO & 0 & 0 & 2 & 0 & 0 \\
\hline SANTA CATALINA & 0 & 0 & 2 & 0 & 1 \\
\hline
\end{tabular}




\begin{tabular}{|l|c|c|c|c|c|}
\hline SANTA ROSA DE LIMA & 0 & 0 & 0 & 0 & 1 \\
\hline SANTA ROSA DEL SUR & 0 & 1 & 2 & 0 & 2 \\
\hline SIMITI & 0 & 1 & 0 & 0 & 0 \\
\hline SOPLAVIENTO & 0 & 0 & 0 & 1 & 0 \\
\hline TALAIGUA NUEVO & 0 & 0 & 0 & 0 & 2 \\
\hline TIQUISIO & 3 & 0 & 0 & 0 & 0 \\
\hline TURBACO & 4 & 3 & 3 & 6 & 2 \\
\hline TURBANA & 0 & 1 & 0 & 0 & 0 \\
\hline VILLANUEVA & 0 & 1 & 0 & 1 & 1 \\
\hline ZAMBRANO & 0 & 0 & 0 & 0 & 0 \\
\hline SIN DATO & 0 & 0 & 0 & 0 & 1 \\
\hline TOTAL & 27 & 23 & 31 & 31 & 32 \\
\hline
\end{tabular}

Fuente: Comité de Validación MCE - COISBOL

\section{PARA DESTACAR:}

- En el periodo objeto de análisis solo se presentaron 29 casos, siendo la tasa de mortalidad por esta causa equivalente a 2,64 por cada cien mil habitantes.

- Son los grandes municipios de Bolívar, en términos poblacionales, los que presentan los mayores casos de suicidios históricamente, encabezados por Magangué, seguido por Turbaco y Carmen de Bolívar.

- El Suicidio es una variable que está más asociada a un problema de salud pública mental, por lo que las acciones para tratarla deben abordarse desde la Secretaría de Salud, y demás instancias de salud con jurisdicción en el departamento.

\section{CONCLUSIONES}

El análisis investigativo aquí consignado ofrece la oportunidad de hacer una revisión y evaluación sobre el desarrollo y ejecución del Plan Integral de Seguridad y Convivencia BOLÍVAR SEGURO, documento que se desprende del Plan Departamental de Desarrollo 2012-2015 BOLÍVAR GANADOR, y que constituye el documento guía y rector de las inversiones en programas y proyectos en materia de seguridad y convivencia. Las inversiones del periodo de gobierno 2012-2015 se reflejaron en áreas como la movilidad, infraestructura, tecnología y comunicaciones y participación comunitaria. El consolidado de todas las inversiones del cuatrienio señala que la cifra global de ejecución del Plan Integral de Seguridad y Convivencia BOLÍVAR SEGURO ha tenido un costo para la Gobernación de Bolívar de \$32.843.388.231. Estas inversiones en seguridad y convivencia, que bien podría ser objeto de otra investigación (análisis y evaluación de Políticas Públicas), generaron un impacto que se evidencia en la reducción de los niveles de inseguridad (medidos a través de la reducción de las muertes por homicidio) en el departamento de Bolívar, y que se traducen en tranquilidad y bienestar social para habitantes y visitantes de la entidad territorial. 


\section{REFERENCIAS BIBLIOGRÁFICAS}

Centro De Observación E Investigación Social De Bolívar. (s.f). Bolivar.gov. Recuperado de: http://www.bolivar.gov.co/index.php/coisbol

Congreso de Colombia. (24 de julio del 2000). Código Penal. [LEY 599 DE 2000].

DANE. (2010). Preguntas relacionadas con las muertes violentas. Preguntas Frecuentes. (pp. 10-11). Recuperado de: https://www.dane.gov.co/files/investigaciones/ poblacion/preguntas_frecuentes.pdf

Gobernación de Bolívar. (26 de junio del 2012). Se adopta el Centro de Observación e Investigación Social de Bolívar (COISBOL). [Decreto 353 de 2012].

Gossaín, J.C. (2014). Plan Integral De Seguridad Y Convivencia "Bolívar Seguro". Recuperado de: file://D:/Users/Usuario/Downloads/1.\%20PLAN\%20INTEGRAL\%20DE\%20 SEGURIDAD\%20Y\%20CONVIVENCIA_ACTUALIZADO\%20\%20\%20\%202014_.pdf

Instituto Nacional de Medicina Legal y Ciencias Forenses. (2014). Forensis 2014. Datos para la vida. Herramienta para la interpretación, intervención y prevención de lesiones de causa externa en Colombia. Recuperado de : http://www.medicinalegal.gov.co/ documents/88730/1656998/Forensis+Interactivo+2014.24-JULpdf.pdf/9085ad79d2a9-4c0d-a17b-f845ab96534b

Ministerio de Salud. (4 de octubre de 1993. Por la cual se establecen las normas científicas, técnicas y administrativas para la investigación en salud. [Resolución número 8430 de 1993]. Recuperado de: https://www.minsalud.gov.co/sites/rid/ Lists/BibliotecaDigital/RIDE/DE/DIJ/RESOLUCION-8430-DE-1993.PDF

Organización Panamericana de la Salud (2009). Informe sobre el estado de la seguridad vial en la región de las Américas. Washington, D.C.: Organización Panamericana de la Salud. Recuperado de: http://www.who.int/violence_injury_prevention/road_ safety_status/2009/gsrrs_paho.pdf

Zapata, P., Betancourt, M. Henao, R., Taborda, G. (2014). Caracterización del suicidio en Risaralda (Colombia), periodo 2008-2012. Revista Colombiana de Medicina Legal y Ciencias Forenses 2(1), 54-60. 